\title{
Time as an operator/observable in nonrelativistic quantum mechanics
}

\author{
G. E. Hahne* \\ NASA, Ames Research Center \\ Moffett Field, California, 94035 USA
}

November 11, 2018

PACS Numbers: 03.65.Ca, 03.65.Xp, 02.30.Jr

\begin{abstract}
The nonrelativistic Schrödinger equation for motion of a structureless particle in four-dimensional space-time entails a well-known expression for the conserved four-vector field of local probability density and current that are associated with a quantum state solution to the equation. Under the physical assumption that each spatial, as well as the temporal, component of this current is observable, the position in time becomes an operator and an observable in that the weighted average value of the time of the particle's crossing of a complete hyperplane can be simply defined: the theory predicts, and experiment is presumed to be able to observe, the integral over the hyperplane of the normal component of probability current, weighted by the time coordinate. In conventional formulations the hyperplane is always spacelike, i.e., is a time $=$ constant hyperplane in Galilean relativity, and the result is then trivial. A nontrivial result is obtained if the plane is not of this type. When the space-time coordinates are $(t, x, y, z)$, the paper analyzes in detail the case that the hyperplane is of the type $z=$ constant. Particles can cross such a hyperplane in either direction, so it proves convenient to introduce an indefinite metric, and correspondingly a sesquilinear inner product with non-Hilbert space structure, for the space of quantum states on such a surface. Since the metric is indefinite, an uncertainty principle involving the dispersion of the crossing time and the dispersion of its conjugate momentum does not appear to be derivable from the theory. A detailed formalism for computing average crossing times on a $z=$ constant hyperplane, and average dwell times and delay times for a zone of interaction contained between a pair of $z=$ constant hyperplanes, is presented.
\end{abstract}

\footnotetext{
*email: hahne@nas.nasa.gov
} 


\section{Introduction}

Within a few years after the discovery of quantum mechanics a consensus formed (von Neumann 1, p. 188 and [2, p. 354, Pauli [3], p. 140, footnote, and 4], p. 63 , footnote) to the effect that, in contrast to spatial positions, and therefore in conflict with special relativity, the temporal position $t$ is necessarily a c-number, or parameter, with no generic operator status being mathematically feasible. In the decades intervening since the publication of the original versions of the two cited treatises in 1932 and 1933, respectively, the prohibition on specifying the time as a dynamical variable has been widely upheld as part of the standard doctrine of quantum mechanics - see, e. g., Peres [5] Chap. 12-7, Omnès 6] p. 57, and Sakurai [7] p. 68. In recent decades interest in this subject has intensified, due in part to applications of tunneling phemomena in semiconductors ( 8 , [9] Ch. 3.2.3, 10, [1], 12, 13]), and a substantial set of results has been published that introduce formalisms that argue for, or against, various quantummechanical definitions of time, including arrival times, tunneling times, dwell (alias sojourn) times, and delay times. For definitions, reviews, and citations, see Refs. 14, 15], 16], 17, [18, [19], and a collection of articles in [20].

This paper claims to advance a general operator for time as a quantummechanical observable in the context of the nonrelativistic Schrödinger equation. A natural preliminary question is, how does the construction of such an observable time square with the putative result that no such operator exists in general? I believe that the nonexistence proof rests on an improper substitution of dynamics for kinematics, and that there is a straightforward interplay between classical and quantum mechanics on this question. We infer from Pauli [3] in a footnote on p. 140, that the search for a classical observable for time reduces to the following problem: Let $\left\{q^{1}, p_{1}, \ldots, q^{N}, p_{N}\right\}$ be the positions and momenta in a generic classical Hamiltonian dynamics, with Hamiltonian $H\left(t, q^{1}, p_{1}, \ldots, q^{N}, p_{N}\right)$. We define the ordinary Poisson bracket $\left\{f_{1}, f_{2}\right\}_{\mathrm{pb}}$ of two functions $f_{1,2}\left(t, q^{1}, p_{1}, \ldots, q^{N}, p_{N}\right)$ to be

$$
\left\{f_{1}, f_{2}\right\}_{\mathrm{pb}}=\sum_{j=1}^{N}\left(\frac{\partial f_{1}}{\partial q^{j}} \frac{\partial f_{2}}{\partial p_{j}}-\frac{\partial f_{1}}{\partial p_{j}} \frac{\partial f_{2}}{\partial q^{j}}\right),
$$

Then we want to find a function $T\left(t, q^{1}, p_{1}, \ldots, q^{N}, p_{N}\right)$ such that

$$
\{T, H\}_{\mathrm{pb}}=1 \text {. }
$$

Such a function $T$ would be the classical limit of a quantum-mechanical Hermitean operator $T_{\mathrm{qm}}$ that is invoked by Pauli, but demonstrated by him not to exist in general, such that the commutator satisfies

$$
\left[T_{\mathrm{qm}}, H_{\mathrm{qm}}\right]=i \hbar 1_{\mathrm{qm}}
$$

Pauli's theorem has prevailed over the intervening decades, and attempts to define an observable time in connection with solutions to the Schrödinger equation have had recourse to many alternate approaches - see the reviews cited above. 
It is easy to see in a related context that Pauli's result, although correct in its limited context, addresses the wrong question. Let us define the augmented Poisson bracket $\left\{F_{1}, F_{2}\right\}_{\mathrm{apb}}$ of two functions $F_{1,2}\left(t, p_{t}, q^{1}, p_{1}, \ldots, q^{N}, p_{N}\right)$ to be

$$
\left\{F_{1}, F_{2}\right\}_{\mathrm{apb}}=\frac{\partial F_{1}}{\partial t} \frac{\partial F_{2}}{\partial p_{t}}-\frac{\partial F_{1}}{\partial p_{t}} \frac{\partial F_{2}}{\partial t}+\left\{F_{1}, F_{2}\right\}_{\mathrm{pb}}
$$

The function $\mathcal{D}_{\mathrm{cl}}$, defined as

$$
\mathcal{D}_{\mathrm{cl}}=p_{t}+H,
$$

generates the time dependence of quantities as $F$, in that

$$
\frac{d F}{d t}=\left\{F, \mathcal{D}_{\mathrm{cl}}\right\}_{\mathrm{apb}},
$$

subject to the dynamical constraint that on classical paths we require

$$
\mathcal{D}_{\mathrm{cl}}=0
$$

(see 21, Ch. VI.10). We now infer that if $F=t$, then $d F / d t=\left\{t, \mathcal{D}_{\mathrm{cl}}\right\}_{\mathrm{apb}}=1$, whatever be the Hamiltonian $H$. In quantum mechanics, with suitable attention to operator ordering, we substitute

$$
\mathcal{D}_{\mathrm{qm}}=\frac{\hbar}{i} \frac{\partial}{\partial t}+H\left(t, q^{1}, \frac{\hbar}{i} \frac{\partial}{\partial q^{1}}, \ldots\right),
$$

and obtain the time-dependent Schrödinger equation for $\psi\left(t, q^{1}, \ldots, q^{N}\right)$ as a dynamical constraint on $\psi$, that is,

$$
\mathcal{D}_{\mathrm{qm}} \psi=0 .
$$

If $\psi$ is a solution to the Schrödinger equation we can substitute $H \psi$ for $i \hbar \partial \psi / \partial t$, but not otherwise. In particular, if $\psi$ is a nontrivial solution to (9), $t \psi$ is not a solution, so that

$$
i \hbar \frac{\partial}{\partial t} t \psi \neq H t \psi
$$

Therefore, trying to satisfy the operator commutation rule (3) is not relevant to the problem of finding an operator for the time, even when the operand is a solution to the Schrödinger equation. However, the general definition for the time-derivative of quantum-mechanical operators

$$
\frac{d F_{\mathrm{qm}}}{d t}=(i \hbar)^{-1}\left[F_{\mathrm{qm}}, \mathcal{D}_{\mathrm{qm}}\right]
$$

does make sense no matter what the state function, dynamically constrained or not, operated on by the rhs: in fact, if $F_{\mathrm{qm}}=t$, whatever be the Hamiltonian, its time derivative operator is the unit operator.

Let the classical Hamiltonian for a particle in four-dimensional space-time be

$$
H=\frac{1}{2 m}\left(p_{x}^{2}+p_{y}^{2}+p_{z}^{2}\right)+V(t, x, y, z) .
$$


Then (7) can be solved for $p_{z}$ to yield

$$
\mathcal{D}_{ \pm \mathrm{cl}}^{\prime}\left(t, p_{t}, x, p_{x}, y, p_{y}, z, p_{z}\right)=p_{z} \mp\left[-2 m\left(p_{t}+V\right)-p_{x}^{2}-p_{y}^{2}\right]^{1 / 2}=0 .
$$

The quantity $\mathcal{D}_{ \pm c l}^{\prime}$, considered as a function of eight variables prior to its role as a constraint, generates the equations of motion for the evolution of the system in the $\pm z$-direction. The same classical dynamics is obtained if we return to the original Lagrangian formalism, take $t$ as a dependent variable and $z$ as the independent variable, and procede to the Hamiltonian formalism. We remark that the quantum operator $\hbar H_{\text {zev }}$ of (24) can be construed as a matrix square root of the quantum operator that arises from a term in (13).

In this paper we shall consider only the nonrelativistic form of quantum mechanics, restricted to the problem of determining the wave function of a massive, structureless particle in Galilean four-dimensional space-time in the presence of a given space- and time-dependent potential energy distribution. The physical hypothesis that underlies the theory herein is that not just the time component, but also the spatial components, of the conserved four-current density of probability flow are observables. This hypothesis does not seem to have been made explicitly, or its consequences studied, heretofore. A sketch of the mathematics introduced to elaborate this physical assumption follows.

We shall treat quantum mechanics as a boundary-value problem for the Schrödinger equation. The specified boundary values on a simple domain in space-time will be regarded as input to the problem, and the derived interior values and complementary boundary values will be considered as the overall output. An intermediate objective will be to formulate a theory of spatial evolution of wave functions. As is usual in applications, we shall choose one spatial coordinate as the evolution coordinate, and expand the wave function in terms of conveniently simple orthogonal functions of the transverse coordinates, which include the time. The time and the two transverse spatial coordinates therefore will appear naturally as operator/observables in the space of such functions, analogous to the role of the three spatial positions when the time is taken as the evolution coordinate.

Suppose that we want to solve the Schrödinger equation in a space-time box surrounded by two $t=$ constant walls and a spatial boundary. Since the equation is of first order in time and of second order in the spatial coordinates, it is, mathematically speaking, necessary and sufficient to supply wave function values on the earlier $t=$ constant surface, and a suitable combination of wave function and normal-derivative values on the spatial boundary surface, to infer that an interior solution exists and is unique, as discussed in [22, Ch. $5, \S 3$. (Our mathematics differs from Friedman's in that his equation is the heat equation rather than the Schrödinger equation, and we shall administer nonlocal boundary conditions, which distinguish input from output signals, on the spatial boundary.) Conventional time-dependent quantum mechanics for the most part deals with specifying initial, or (but not and) occasionally final, values on a $t=$ constant surface and simple (often, zero) values on the spatial boundary, which can be partly or wholly at infinity. Nontrivial spatial boundary values, 
as incoming wave amplitudes in a scattering problem, are conventionally specified only in the context of the time-independent Schrödinger equation. In the present work we shall generalize the latter problem by considering general timedependent, as well as space-dependent, input values on the spatial boundary, in the presence of explicit time dependence in the potential energy function in the differential equation itself. We shall hereinafter denote these cases of boundary value problems as Type I and Type II, respectively. These correspond, roughly and respectively, to the first and second initial-boundary value problems analyzed in 22 , Chs. 3 and 5 .

In the first problem, the wave function evolves in time from given initial values, with time-independent spatial boundary values. In the second problem, we shall consider that the wave function evolves with respect to the $z$-coordinate, such that the interior domain corresponds to a finite interval in the chosen coordinate $z$. Since the differential equation is of second order, determining the evolution of a wave function in a spatial direction is generally a more difficult task of analysis in both the mathematical and physical senses than one for its evolution in time. We summarize the derivation to be carried out below in terms of the following ten observations, steps, or results: (i) the space of states on any given $z=z_{1}$ hyperplane has a natural doubled structure in that it comprises the direct sum of the values and of the $z$-derivatives of the usual space of wave functions $\psi(t, x, y, z)$ at $z=z_{1}$; (ii) the Hamiltonian is a $2 \times 2$ matrix of operators that is derived from the ordinary Schrödinger equation; (iii) the familiar expression for the probability current density in the $z$-direction is used to infer the definition of a metric operator in the space of states, where now inner products include an integral over $t$ as well as over $x$ and $y$; (iv) the metric so derived is indefinite, and the Hamiltonian is self-adjoint with respect to the metric (synonymously, pseudo-Hermitean); (v) the norm being indefinite, we shall sometimes use the term "particle presence" to denote the unit operator, the expectation value of which is the above-mentioned norm; (vi) apart from modifications needed for closed channels, the formalism can be established so that waves traveling in the $+z$ direction have positive norm, and waves traveling in the $-z$ direction have negative norm with respect to the metric; (vii) while for open-channel modes the direction of travel and of propagation will coincide in the large, it is convenient to define these categories differently for closed-channel modes; (viii) the input and output at either end of a finite spatial interval $\left[z_{1}, z_{2}\right]$ are taken to comprise, respectively, the superposition of waves propagating into, and the superposition of waves propagating out of, the interval at the initial point $z_{1}$ and at the final point $z_{2}$ (this means that there will be only outward propagating scattered waves from a zone of interaction); (ix) orthonormal sets of input or of output states, transition amplitudes, and probabilities are then computed using what amounts to a Hilbert space inner product, which is derived from the indefinite metric, but depends on the wave function and its $z$-derivative at both $z_{1}$ and $z_{2} ;(\mathrm{x})$ the dynamics yields a mapping of open-channel input into open-channel output that is unitary.

The fact that a pseudo-Hermitean Hamiltonian describes the spatial evolution of a physical system's wave function has another concomitant: the Hamil- 
tonian can have, as well as real eigenvalues, nonreal eigenvalues that occur in complex conjugate pairs (Gohberg, et al., 23] p. 23, Proposition 2.4). Each such pair is associated with the two wave function solutions (one rising, the other falling exponentially) for a closed channel, or classically inacessible region for the system when it is in an associated quantum state. We shall argue that it is natural to define the direction of propagation (but not of travel of the particle that the wave represents - see the discussion in Sec. 4) of such a wave as the direction in which it decreases exponentially. The simple exponential states in such a pair each have zero norm and, with proper normalization, unit overlap, which complicates the formalism. A further complication results from the circumstance that a degenerate eigenvalue of a Hamiltonian requires special treatment when the Hamiltonian cannot be diagonalized by a similarity transformation, leading to the appearance of so-called " $n$-pole ghost" quantum states, for $n=2,3, \ldots$. There is more discussion on these problems below.

Formalisms for the spatial evolution of a wave function were proposed by Kijowski [24] and by Piron [25], and their work was discussed by Mielnik [26]. These two approaches differ substantially from each other and from the formalism introduced herein, as will be discussed following Eq. (24) and in Sec. 4 .

The quantum mechanics describing evolution of a wave function in both directions across a spatial interval is to an extent patterned after the author's previous work [27] on a quantum dynamics that encompasses joint bidirectional evolution of a quantum state between two temporal walls.

The remaining sections are organized as follows: In Sec. 2, we shall formulate expressions for the four-current density associated with a physical quantity, and for the local space-time density for creating or destroying that quantity in a quantum-mechanical system. We shall also show how to prescribe physically motivated boundary conditions so that the Schrödinger equation can be solved in a semi-infinite (finite in the $z$-direction, infinite in the $t, x, y$-directions) box. In Section 3 we shall propose a formalism for computing the average temporal position (i.e., crossing time) of the particle at both spatial walls of the box, given the spatial input and given the $S$-matrix deriving from a general interaction potential energy in the box's interior. These results will then be used to compute formulas for dwell and delay times for the particle remaining within, reflecting from, or transmitted across, the box. Section 4 concludes the paper with a discussion of the present formalism and of previous work on the subject. The matters of an uncertainty principle involving the time and its conjugate momentum, and of an extended theory of measurement, are merely touched on there, as attempts by the author to develop these constructs have not been successful.

\section{Quantum-mechanical formalism}

In this section we shall set up the theory that forms the "floor" of the present work. Rather than attempt to make the formalism highly general, we shall 
develop the argument in a particular context: the wave function solution of Schrödinger's equation for a particle moving in the interior of a certain simple box of four-dimensional space-time. In particular, we propose a formalism and an interpretation that incorporate the wave function into an expression for the space-time four-vector "flow" density of a physical quantity, which quantity corresponds to a certain linear operator in the function space of fully time- and space-dependent wave functions. We shall argue that is is natural to regard the four-divergence of the flow as the local density of creation and destruction of that quantity at a point in space-time for the physical system in that timedependent quantum state. Either the volume integral of the divergence, or the surface integral of the normal component of the flow vector density, therefore represents the total amount of that quantity generated inside the space-time box. If that quantity is the time $t$, this integral plausibly represents the average so-called dwell time of the particle in the given box, given that the wave function is properly normalized.

Let $\mathcal{B}_{1}$ and $\mathcal{B}_{2}$ be the following open boxes in space-time:

$$
\begin{aligned}
& \mathcal{B}_{1}=\left\{(t, x, y, z) \mid t_{1}<t<t_{2},-\infty<x<\infty,-\infty<y<\infty,-\infty<z<\infty\right\} . \\
& \mathcal{B}_{2}=\left\{(t, x, y, z) \mid-\infty<t<\infty,-\infty<x<\infty,-\infty<y<\infty, z_{1}<z<z_{2}\right\} .
\end{aligned}
$$

The Schrödinger equation for $\psi(t, x, y, z)$ for a particle of mass $m$ can be derived from a variational principle for an action $\mathcal{A}$, as given in $\operatorname{Schiff}(28$, p. 499), but modified to make it real:

$\mathcal{A}=\iiint \int_{\mathcal{B}} d t d x d y d z\left[\frac{i \hbar}{2} \psi^{*} \frac{\partial \psi}{\partial t}-\frac{i \hbar}{2} \frac{\partial \psi^{*}}{\partial t} \psi-\frac{\hbar^{2}}{2 m} \nabla \psi^{*} \cdot \nabla \psi-\psi^{*} V(t, x, y, z) \psi\right]$.

The equations of motion are to be obtained by keeping the boundary values of $\psi$ and $\psi^{*}$ fixed, and pretending that in the interior region $\psi$ and $\psi^{*}$ can be varied independently and "arbitrarily". The action is stationary when $\psi(t, x, y, z)$ satisfies

$$
\frac{\hbar}{i} \frac{\partial \psi}{\partial t}-\frac{\hbar^{2}}{2 m} \nabla^{2} \psi+V(t, x, y, z) \psi=0,
$$

and $\psi(t, x, y, z)^{*}$ satisfies the complex conjugate equation, for all $(t, x, y, z) \in$ $\mathcal{B}_{1}$ or $\mathcal{B}_{2}$.

Henceforth when we say "solution", we shall mean a function defined over the entire box such that it satisfies equation (16) everywhere in $\mathcal{B}_{1}$ or $\mathcal{B}_{2}$. The linear operators representing physical quantities will normally carry a solution into another space- and time-dependent function that is not a solution, so in effect we shall deal with the more general vector space of well-behaved, complexvalued functions of space and time that need not be solutions of the Schrödinger equation.

A standard problem in conventional quantum mechanics arises if we constrain $\psi(t, x, y, z)$ to be zero on the infinite parts of the spatial boundary of the 
box $\mathcal{B}_{1}$, that is

$$
\psi(t, x, y, z) \rightarrow 0, \text { if }|x|+|y|+|z| \rightarrow \infty
$$

and require that

$$
\psi(t, x, y, z) \rightarrow u(x, y, z), \quad \text { as } t \rightarrow t_{1},
$$

where $u(x, y, z)$ is some given complex-valued function on the earlier temporal boundary of the box. As is well known, the interior values of $\psi(t, x, y, z)$, and the limiting values on the temporally later boundary of the box at $t=t_{2}$, are all uniquely determined by the differential equation and these input boundary conditions. The derived values are all output in a sense, but we shall often mean by output just the subset of boundary values that were not given as input, in the present case $\psi\left(t_{2}, x, y, z\right)$ with $-\infty<x<\infty,-\infty<y<\infty$, and $-\infty<z<\infty$.

Now let us consider a problem in box $\mathcal{B}_{2}$ such that certain information about the limiting values of $\psi(t, x, y, z)$ and $\partial \psi / \partial z(t, x, y, z)$ on the two walls $z=z_{1}$ and $z=z_{2}$ is given as input, while the wave function is supposed to tend to zero as $t$ and/or $x$ and/or $y$ tend to $\pm \infty$. We want to specify just enough input information so that a solution satisfying the input boundary conditions exists and is unique. In order to accomplish this, we need to do some preliminary work. We shall not keep to a mathematically rigorous derivation, but appeal to plausibility arguments at most steps.

We now convert the above variational principle to Hamiltonian form using the methods of Goldstein (29], Chap. 12-4), with the proviso that it is the spatial parameter $z$, rather than $t$, that is taken as the evolution coordinate for the wave function. The quantity in square brackets in (15) is the Lagrangian density $\mathcal{L}$. The canonical field momenta are

$$
\begin{aligned}
p_{\psi} & =\frac{\partial \mathcal{L}}{\partial\left(\frac{\partial \psi}{\partial z}\right)}=-\frac{\hbar^{2}}{2 m} \frac{\partial \psi^{*}}{\partial z}, \\
p_{\psi^{*}} & =\frac{\partial \mathcal{L}}{\partial\left(\frac{\partial \psi^{*}}{\partial z}\right)}=-\frac{\hbar^{2}}{2 m} \frac{\partial \psi}{\partial z} .
\end{aligned}
$$

The action functional becomes

$$
\mathcal{A}=\iiint \int_{\mathcal{B}_{2}} d t d x d y d z\left[p_{\psi} \frac{\partial \psi}{\partial z}+\frac{\partial \psi^{*}}{\partial z} p_{\psi^{*}}-\mathcal{H}\left(\psi, p_{\psi}, \psi^{*}, p_{\psi^{*}}\right)\right],
$$

where the Hamiltonian density is

$$
\mathcal{H}=-\frac{2 m}{\hbar^{2}} p_{\psi} p_{\psi^{*}}-\frac{i \hbar}{2} \psi^{*} \frac{\partial \psi}{\partial t}+\frac{i \hbar}{2} \frac{\partial \psi^{*}}{\partial t} \psi+\frac{\hbar^{2}}{2 m}\left(\frac{\partial \psi^{*}}{\partial x} \frac{\partial \psi}{\partial x}+\frac{\partial \psi^{*}}{\partial y} \frac{\partial \psi}{\partial y}\right)+\psi^{*} V \psi
$$

The equations of motion obtained by varying $\psi^{*}$ and $p_{\psi}$ are a coupled set of linear equations; a complex conjugate set is obtained by varying $\psi$ and $p_{\psi^{*}}$. We write the former equations in $2 \times 2$ matrix-operator form as follows: We first define

$$
\Psi(t, x, y, z)=\left[\begin{array}{c}
\psi(t, x, y, z) \\
p_{\psi^{*}}(t, x, y, z)
\end{array}\right]
$$


then the equations of motion can be written

$$
\frac{1}{i} \frac{\partial \Psi}{\partial z}=H_{\mathrm{zev}} \Psi
$$

where the Hamiltonian $H_{\text {zev }}$ (the subscript "zev" stands for "z-evolution") is

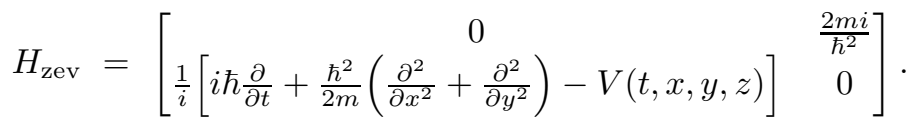

We note that Piron [25] obtained a Schrödinger equation for a wave function's evolution along the spatial coordinate $x$, but Piron's wave function has one component, and the Hamiltonian is the operator derived from the classical quantity that generates dynamical motion along a the $x$-axis. Piron thereupon obtained a general expression for the evolution in $x$ of the average temporal position of a particle in one space dimension, but did not develop the theory further.

In the ordinary quantum mechanics derivable from the variational principle Eq. (15), the $z$-component of the conserved probability four-current density is (28, p. 27)

$$
J_{3}(t, x, y, z)=\frac{\hbar}{2 i m}\left(\psi^{*} \frac{\partial \psi}{\partial z}-\frac{\partial \psi^{*}}{\partial z} \psi\right) .
$$

In the present language this expression takes the form

$$
J_{3}=\hbar^{-1} \Psi^{\dagger} M \Psi
$$

where $M$ is the $2 \times 2$ matrix

$$
M=\left[\begin{array}{cc}
0 & i \\
-i & 0
\end{array}\right]
$$

Note that the matrix $M$ is Hermitean, has unit square, and has eigenvalues \pm 1 , so that it can engender an indefinite metric. By inference, we make a guess for an inner product law for two $z$-propagating states:

$$
\left(\Psi_{1}(z) ; \Psi_{2}(z)\right)=\hbar^{-1} \iiint_{\mathbb{R}^{3}} d t d x d y\left[\Psi_{1}(t, x, y, z)^{\dagger} M \Psi_{2}(t, x, y, z)\right] .
$$

Note that this formula has the appropriate physical dimensions, in that if $\psi_{1,2}$ have the usual dimension length ${ }^{-3 / 2}$, then the above inner product is dimensionless.

We shall now argue that the above ingredients can be made into a theory of spatial evolution of a Schrödinger wave function. We shall work with the case of $z$-evolution of a wave function in four-dimensional space-time, but generalizations to other cases, as radial or reaction coordinates (see [30]) for the $(3 N+1)$-dimensional space-time involved in an $N$-particle wave function, are formally straighforward. 
Let $\mathcal{S}$ be the space of functions of type Eq. (22), with some appropriate boundary conditions. We define the $M$-adjoint of a linear operator $W$ acting on this space as that unique operator $W^{\ddagger}$ such that

$$
\left(W^{\ddagger} \Psi_{1} ; \Psi_{2}\right)=\left(\Psi_{1} ; W \Psi_{2}\right)
$$

for all $\Psi_{1}, \Psi_{2} \in \mathcal{S}$; in $2 \times 2$ matrix form, with $W^{\dagger}$ as the ordinary Hermitean conjugate, we have

$$
W^{\ddagger}=M W^{\dagger} M \text {. }
$$

If

$$
W^{\ddagger}=W
$$

we call $W$ pseudo-Hermitean, and if

$$
W^{\ddagger}=W^{-1}
$$

we call $W$ pseudo-unitary.

If $W$ is pseudo-Hermitean and the state $\Psi$ is suitably normalized, we want to make the plausible specification that the (necessarily real) number $(\Psi ; W \Psi)$ is the expectation value of $W$ in the state $\Psi$. We argue in favor of this axiom as follows: Let $x^{0}=t, x^{1}=x$, and so on. Suppose that in the conventional Schrödinger formalism, $\omega$ is some physical quantity, such as the time $\check{t}$, the spatial positions $\check{x}, \check{y}, \check{z}$, or the "particle presence" $\breve{1}$ (we denote operators standing for physical parameters with a "haček" accent over the symbols). We take as a physical axiom that the four-vector "flow" density $J_{\mu}^{(\omega)}(t, x, y, z), \mu=0,1,2,3$, of $\omega$ is

$$
\begin{aligned}
J_{0}^{(\omega)}(t, x, y, z) & =\psi(t, x, y, z)^{*} \omega \psi(t, x, y, z) \\
J_{k}^{(\omega)}(t, x, y, z) & =\frac{\hbar}{2 i m}\left(\psi(t, x, y, z)^{*} \omega \frac{\partial \psi}{\partial x^{k}}(t, x, y, z)\right. \\
& \left.-\frac{\partial \psi^{*}}{\partial x^{k}}(t, x, y, z) \omega \psi(t, x, y, z)\right), \text { for } k=1,2,3 .
\end{aligned}
$$

The above expressions need symmetrization if $\omega$ contains derivative operators, e.g., $\omega=X_{\mathrm{cm}}^{j}=x^{j}+t(i \hbar / m) \partial / \partial x^{j}$, one of the components of the initial centerof-mass position for a free particle. We compute the four-divergence of the above vector field, assuming that $\psi$ is a solution to Eq. (16):

$$
\begin{aligned}
\sum_{\mu=0}^{3} \frac{\partial J_{\mu}^{(\omega)}}{\partial x^{\mu}}(t, x, y, z) & =\frac{1}{i \hbar} \psi^{*}[\omega, V] \psi+\psi^{*} \frac{\partial \omega}{\partial t} \psi \\
& +\frac{\hbar}{2 i m} \sum_{k=1}^{3}\left(\psi^{*} \frac{\partial \omega}{\partial x^{k}} \frac{\partial \psi}{\partial x^{k}}-\frac{\partial \psi^{*}}{\partial x^{k}} \frac{\partial \omega}{\partial x^{k}} \psi\right) .
\end{aligned}
$$

This divergence can be construed to be the local density of creation or destruction of the quantity $\omega$ by the system in the state $\psi(t, x, y, z)$. If the divergence is zero, as for the case $\omega=\check{1}$, the associated quantity is not being created 
or destroyed and is both globally and locally conserved. If $\omega=\check{t}$, we find that

$$
\sum_{\mu=0}^{3} \frac{\partial J_{\mu}^{(\breve{t})}}{\partial x^{\mu}}(t, x, y, z)=\psi^{*} \psi .
$$

Hence, the so-called "dwell" time $\tau_{D}$ of the particle in a box $\mathcal{B}$ with the given input is the space-time integral of the density of creation of time over the box, that is,

$$
\tau_{D}=\iiint \int_{\mathcal{B}} d t d x d y d z|\psi(t, x, y, z)|^{2} .
$$

The latter result reproduces a formula given in Ref. 17. Eq. (2.2), Ref. 31 Eq. (2.67), and Ref. 32] Eq. (14). By the divergence theorem we can convert the volume integral to a surface integral, so that we have either

$$
\begin{aligned}
\tau_{D}^{I} & =\left.\left[\iiint_{\mathbb{R}^{3}} d x d y d z J_{0}^{(\check{t})}(t, x, y, z)\right]\right|_{t=t_{1}} ^{t=t_{2}}, \text { or } \\
\tau_{D}^{I I} & =\left.\left[\iiint_{\mathbb{R}^{3}} d t d x d y J_{3}^{(\check{t})}(t, x, y, z)\right]\right|_{z=z_{1}} ^{z=z_{2}} .
\end{aligned}
$$

Hence if we have a boundary problem of Type I, such that $\psi(t, x, y, z)$ is zero on the spatial walls, and $\psi$ has the usual conserved unit norm on the $t=$ constant walls, we find, with Eq. 33a

$$
\tau_{D}^{I}=t_{2}-t_{1} .
$$

If we have a boundary value problem of Type II - we shall discuss later how to normalize $\psi$ in that case - so that $\psi(t, x, y, z)$ tends to zero as $|t|$ or $|x|$ or $|y|$ becomes large, and using Eq. (33b) then

$$
\begin{aligned}
\tau_{D}^{I I} & =\left[\frac { \hbar } { 2 i m } \int \int \int _ { \mathbb { R } ^ { 3 } } d t d x d y \left(\psi(t, x, y, z)^{*} t \frac{\partial \psi}{\partial z}(t, x, y, z)\right.\right. \\
& \left.\left.-\frac{\partial \psi^{*}}{\partial z}(t, x, y, z) t \psi(t, x, y, z)\right)\right]\left.\right|_{z=z_{1}} ^{z=z_{2}} .
\end{aligned}
$$

The alternate forms of the dwell time given in Eqs. (36) and (39) were previously obtained by Jaworski and Wardlaw and applied in a series of papers (33. Eqs. (4.2) and (A1), 34, 35], [36]). Given that we compute $\Psi(t, x, y, z)$ from $\psi(t, x, y, z)$ by Eq. (22), and that $I_{2}$ is the $2 \times 2$ unit matrix, then the operator $\check{t} I_{2}$ is the time operator in the $z$-evolution formalism, and we have, in the notation of Eq. (28),

$$
\tau_{D}^{I I}=\left.\left(\Psi(z) ;\left(\check{t} I_{2}\right) \Psi(z)\right)\right|_{z=z_{1}} ^{z=z_{2}} .
$$

The above results suggest that for a pseudo-Hermitean operator $W$ in the space of $\Psi$-solutions, and for a boundary value problem of Type II, we should 
define the expectation value $\langle W\rangle_{\Psi(z)}$ of $W$ in the state $\Psi(t, x, y, z)$ at a chosen $z$ as

$$
\langle W\rangle_{\Psi(z)}=(\Psi(z) ; W \Psi(z)),
$$

as was proposed earlier in this section. The value $\langle W\rangle_{\Psi(z)}$ therefore (in Type II problems) specifies the average net flow of $W$ across the given $z=$ constant surface. The difference of the expectation values of $W$ computed at $z=z_{2}$ and $z=z_{1}$ is therefore the net flow of $W$ out of the box, in other words is, on average, the total amount of $W$ "created" by the system in the box.

We want now to define input and output on the spatial walls of the box. We define a complete, orthonormal basis $\phi_{\left(k_{t}, k_{x}, k_{y}\right)}(t, x, y)$ for all $(t, x, y) \in \mathbb{R}^{3}$ as follows:

$$
\phi_{\left(k_{t}, k_{x}, k_{y}\right)}(t, x, y)=(2 \pi)^{-3 / 2} \exp \left(-i k_{t} t+i k_{x} x+i k_{y} y\right)
$$

where $k_{t}, k_{x}$, and $k_{y}$ each range independently from $-\infty$ to $+\infty$. Although the physical dimension of $k_{t}$ differs from that of $k_{x}$ and $k_{y}$, it is convenient to use three-vector notation $\mathbf{k}=\left(k_{t}, k_{x}, k_{y}\right)$ and call the three-volume element $d^{3} k=$ $d k_{t} d k_{x} d k_{y}$. The negative sign before $k_{t}$ in the exponent in Eq. (42) is chosen so that positive $k_{t}$ corresponds to positive energy; the conjugate momentum to $t$ is $p_{t} \leftrightarrow(\hbar / i) \partial / \partial t \leftrightarrow-\hbar k_{t}$.

In an expansion of a wave function $\Psi(t, x, y, z)$ in the above basis functions, we will encounter certain quantities repeatedly, so we now define simplified notation for them: Let $\zeta$ take either value $F$ or $B$, which stand for forward and backward propagation along $z$, respectively. We also take

$$
\sigma(\zeta)= \begin{cases}+1 & \text { if } \zeta=F \\ -1 & \text { if } \zeta=B\end{cases}
$$

If $\left(2 m k_{t} / \hbar\right)>\left(k_{x}^{2}+k_{y}^{2}\right)$ (called an open channel), we define

$$
k_{z}(\mathbf{k})=\left[2 m k_{t} / \hbar-k_{x}^{2}-k_{y}^{2}\right]^{1 / 2},
$$

and if $\left(2 m k_{t} / \hbar\right)<\left(k_{x}^{2}+k_{y}^{2}\right)$ (called a closed channel), we define

$$
\kappa_{z}(\mathbf{k})=\left[-2 m k_{t} / \hbar+k_{x}^{2}+k_{y}^{2}\right]^{1 / 2} .
$$

We shall normally just use $k_{z}$ and $\kappa_{z}$ without explicitly citing their arguments, except that primed, double primed, and triple primed arguments will be denoted, respectively, by $k_{z}^{\prime}, k_{z}^{\prime \prime}$, and $k_{z}^{\prime \prime \prime}$, and similarly for $\kappa_{z}$.

Let a wave function have the expansion in basis functions

$$
\Psi(t, x, y, z)=\iiint_{\mathbb{R}^{3}} d^{3} k \sum_{\zeta=B}^{F} f^{\zeta}(\mathbf{k}) \phi_{\mathbf{k}}(t, x, y) X^{\zeta}(\mathbf{k} ; z),
$$

where the $f^{\zeta}(\mathbf{k})$ are the expansion amplitudes, and where the $X^{\zeta}(\mathbf{k} ; z)$ are normalized solutions for forward or backward motion along $z$, which we construct 
as follows: Substituting Eq. (46) into Eq. (23), we find that

$$
\frac{1}{i} \frac{d X^{\zeta}}{d z}(\mathbf{k} ; z)=H_{\mathrm{zev}}(\mathbf{k} ; z) X^{\zeta}(\mathbf{k} ; z),
$$

where, for $V$ a function of $z$ alone,

$$
H_{\mathrm{zev}}(\mathbf{k} ; z)=\left[\begin{array}{cc}
0 & 2 m i / \hbar^{2} \\
\left(\hbar^{2} / 2 m i\right)\left[2 m k_{t} / \hbar-k_{x}^{2}-k_{y}^{2}-2 m V(z) / \hbar^{2}\right] & 0
\end{array}\right] .
$$

When $V(z) \equiv 0$, and for open channels, we obtain the solutions

$$
X^{\zeta}(\mathbf{k} ; z)=\left[\begin{array}{c}
{\left[m /\left(\hbar k_{z}\right)\right]^{1 / 2} \exp \left[\sigma(\zeta) i k_{z} z\right]} \\
-\sigma(\zeta)(i / 2)\left(\hbar^{3} k_{z} / m\right)^{1 / 2} \exp \left[\sigma(\zeta) i k_{z} z\right]
\end{array}\right]
$$

the corresponding inner products are independent of $z$ :

$$
\hbar^{-1} X^{\zeta^{\prime}}(\mathbf{k} ; z)^{\dagger} M X^{\zeta}(\mathbf{k} ; z)=\delta^{\zeta^{\prime} \zeta} \sigma(\zeta) .
$$

Note, however, that these solutions do not satisfy the Cauchy inequality, in that $\left|\hbar^{-1} X^{\zeta}\left(\mathbf{k}^{\prime}, z\right)^{\dagger} M X^{\zeta}(\mathbf{k}, z)\right|=(1 / 2)\left(\sqrt{k_{z}^{\prime} / k_{z}}+\sqrt{k_{z} / k_{z}^{\prime}}\right)$, which is greater than 1 unless $k_{z}^{\prime}=k_{z}$. For closed channels the solutions are

$$
X^{\zeta}(\mathbf{k} ; z)=\left[\begin{array}{c}
{\left[m /\left(\hbar \kappa_{z}\right)\right]^{1 / 2} \exp \left[-\sigma(\zeta)\left(i \pi / 4+\kappa_{z} z\right)\right]} \\
\sigma(\zeta)(1 / 2)\left(\hbar^{3} \kappa_{z} / m\right)^{1 / 2} \exp \left[-\sigma(\zeta)\left(i \pi / 4+\kappa_{z} z\right)\right]
\end{array}\right]
$$

the inner products take the $z$-independent forms

$$
\hbar^{-1} X^{\zeta^{\prime}}(\mathbf{k} ; z)^{\dagger} M X^{\zeta}(\mathbf{k} ; z)=\delta^{\zeta^{\prime} F} \delta^{B \zeta}+\delta^{\zeta^{\prime} B} \delta^{F \zeta} .
$$

In general, the properties that distinguish between between the four types of state of motion of a particle, that is open- versus closed-channel type, and $F$ versus $B$ type, depend on the local behavior of the state vector in wavenumber space $\left(k_{t}, k_{x}, k_{y}\right)$. The corresponding position $(t, x, y)$ space forms of these properties are nonlocal. As mentioned in Section 4, these properties are likely to complicate an attempt to make a physical interpretation, in the context of the present formalism, of measurements at a given $z$ of local properties in position $t, x$, or $y$. This is in contrast to standard quantum mechanics with $t$ as the evolution coordinate, where there is only one type of state in $x, y, z$ : $F$-type and open channel.

We note that the intermediate free-particle case $2 m k_{t} / \hbar=k_{x}^{2}+k_{y}^{2}$ gives rise to a "dipole ghost" state, in that the reduced Hamiltonian on the rhs of Eq. (48) cannot be diagonalized by a similarity transformation. The construct, which Heisenberg named (see 37, references given therein, and [38], p. 14), derives from the definition of the minimal polynomial of a finite-dimensional, square, complex matrix - see MacLane and Birkhoff [39, Ch. IX.6: Let $L$ be a pseudo-Hermitean operator such that there exists a (real or nonreal) eigenvalue $\lambda$ of $L$, an integer $n \geq 2$, and a state $X_{\lambda n}$ so that the state $(L-\lambda)^{n-1} X_{\lambda n}$ is not the zero state and is an eigenstate in that $(L-\lambda)^{n} X_{\lambda n}=0$, then $X_{\lambda n}$ 
will be called an "n-pole ghost" state of $L$ associated with the eigenvalue $\lambda$. We presume that, for any given $L$, and for each of its eigenvalues $\lambda$, there is a bounded number - possibly zero - of types of ghosts associated with it. The ghost states associated with fixed $L$ and $\lambda$, and of different pole-orders $n$ and $m$, are linearly independent of each other and of associated eigenstates; the direct sum of all the eigenstates and of all the corresponding linearly independent ghost states is a complete set of states in the overall space.

Continuing with the zero-potential-energy, intermediate-case solutions, we note that the symbols $\mathrm{F}$ and $\mathrm{B}$ are not useful. We take the solutions $X^{\alpha}\left(\hbar\left(k_{x}^{2}+\right.\right.$ $\left.\left.k_{y}^{2}\right) /(2 m), k_{x}, k_{y} ; z\right)$ where $\alpha=1,2$, as follows:

$$
\begin{aligned}
X^{1}\left(\hbar\left(k_{x}^{2}+k_{y}^{2}\right) /(2 m), k_{x}, k_{y} ; z\right) & =\left[\begin{array}{c}
{[m \rho / \hbar]^{1 / 2}} \\
0
\end{array}\right], \\
X^{2}\left(\hbar\left(k_{x}^{2}+k_{y}^{2}\right) /(2 m), k_{x}, k_{y} ; z\right) & =\left[\begin{array}{l}
{[m /(\rho \hbar)]^{1 / 2} 2 i z} \\
-i\left[\hbar^{3} /(m \rho)\right]^{1 / 2}
\end{array}\right],
\end{aligned}
$$

where $\rho$ is an arbitrary positive number of dimension length introduced to make the components dimensionally consistent with Eqs. (49) and (51). The inner products are also $z$-independent:

$$
\begin{aligned}
\hbar^{-1} X^{\alpha^{\prime}}\left(\hbar\left(k_{x}^{2}+k_{y}^{2}\right) /(2 m), k_{x}, k_{y} ; z\right)^{\dagger} M X^{\alpha}\left(\hbar\left(k_{x}^{2}+k_{y}^{2}\right) /(2 m), k_{x}, k_{y} ; z\right) \\
= \begin{cases}0, & \text { if } \alpha^{\prime}=\alpha, \\
+1, & \text { if } \alpha^{\prime} \neq \alpha .\end{cases}
\end{aligned}
$$

Physically, the states in the intermediate case propagate parallel to any plane $z=$ constant, that is, neither forward nor backward along $z$. Note that the solution Eq. (53a) is, and that of Eq. (53b) is not, an eigenstate with eigenvalue zero of the reduced Hamiltonian on the rhs of Eq. (48); in fact, the $X^{2}$ is a dipole ghost state for any choice of $k_{x}, k_{y}$ and $z$.

We next compute the inner product at each $z$ of two free-particle wave functions $\Psi(t, x, y, z)$ and $\Phi(t, x, y, z)$, when they have expansion amplitudes $f^{\zeta}(\mathbf{k})$ and $g^{\zeta}(\mathbf{k})$, respectively. It is convenient to divide $\mathbf{k}$-space into domains for open and closed channels:

$$
\begin{aligned}
\iiint_{\text {open }} d^{3} k & =\iint_{\mathbb{R}^{2}} d k_{x} d k_{y} \int_{\hbar\left(k_{x}^{2}+k_{y}^{2}\right) / 2 m}^{\infty} d k_{t}, \\
\iiint_{\text {closed }} d^{3} k & =\iint_{\mathbb{R}^{2}} d k_{x} d k_{y} \int_{-\infty}^{\hbar\left(k_{x}^{2}+k_{y}^{2}\right) / 2 m} d k_{t}, \\
\iiint_{\mathbb{R}^{3}} d^{3} k & =\iiint_{\text {open }} d^{3} k+\iiint_{\text {closed }} d^{3} k .
\end{aligned}
$$


The inner product of $\Psi$ and $\Phi$ is $z$-independent, and takes the form

$$
\begin{aligned}
(\Psi(z) ; \Phi(z)) & =\iiint_{\text {open }} d^{3} k \sum_{\zeta=B}^{F} \sigma(\zeta) f^{\zeta}(\mathbf{k})^{*} g^{\zeta}(\mathbf{k}) \\
& +\iiint_{\text {closed }} d^{3} k\left[f^{F}(\mathbf{k})^{*} g^{B}(\mathbf{k})+f^{B}(\mathbf{k})^{*} g^{F}(\mathbf{k})\right] .
\end{aligned}
$$

Note that the subspace generated by " $F$ " open-channel states has a positive definite norm, while the space of " $B$ " open-channel states has a negative definite norm; each of these subspaces therefore comprises a Hilbert space. In the scattering phenomena analysed in Section 3 we shall discover that the openchannel sub-matrix of the $S$-matrix is unitary, and preserves the inner product of two vectors belonging to a direct sum of these Hilbert spaces referring to different $z$-planes, assembled so that the sign of the inner product and metric are reversed in the second subspace component-hence there is a positive definite metric overall. The " $F$ " and " $B$ " Hilbert spaces on any $z=$ constant plane are of limited utility, as most linear operators encountered in the space of states do not map such a Hilbert space into itself, but generate superpositions of $F$ - and $B$-states, and of open- and closed-channel states.

The question of normalizing the space-evolving wave functions can now be addressed: If the potential $V(t, x, y, z) \neq 0$ in, and only in, the interior of the box $\mathcal{B}_{2}$, a solution $\Psi(t, x, y, z)$ of Eq. (23) can be expressed in an expansion of the type Eq. (46), belonging to potential-free regions, in the neighborhood of both $z=z_{1}$ and $z=z_{2}$, but with different expansion amplitudes at each end of the interval. We first define the basis functions

$$
\Xi^{\zeta}(\mathbf{k} ; t, x, y, z)=(2 \pi)^{-3 / 2} \exp \left(-i k_{t} t+i k_{x} x+i k_{y} y\right) X^{\zeta}(\mathbf{k} ; z) .
$$

We assume here and unless otherwise stated that there is no closed-channel input, and adopt the following conventions:

$$
\begin{aligned}
\Psi\left(t, x, y, z_{1}\right)= & \iiint_{\text {open }} d^{3} k f_{\text {in }}^{F}(\mathbf{k}) \Xi^{F}\left(\mathbf{k} ; t, x, y, z_{1}\right) \\
& +\iiint_{\mathbb{R}^{3}} d^{3} k f_{\text {out }}^{B}(\mathbf{k}) \Xi^{B}\left(\mathbf{k} ; t, x, y, z_{1}\right), \\
\Psi\left(t, x, y, z_{2}\right)= & \iiint_{\text {open }} d^{3} k f_{\text {in }}^{B}(\mathbf{k}) \Xi^{B}\left(\mathbf{k} ; t, x, y, z_{2}\right) \\
& +\iiint_{\mathbb{R}^{3}} d^{3} k f_{\text {out }}^{F}(\mathbf{k}) \Xi^{F}\left(\mathbf{k} ; t, x, y, z_{2}\right),
\end{aligned}
$$

Since the flow of particle presence is conserved, the norms of $\Psi\left(t, x, y, z_{1}\right)$ and $\Psi\left(t, x, y, z_{2}\right)$ are equal:

$$
\langle\check{1}\rangle_{\Psi\left(z_{2}\right)}=\langle\check{1}\rangle_{\Psi\left(z_{1}\right)} .
$$

Note that at $z=z_{1}$, the forward and backward propagating parts of the wave function correspond to input and output, respectively, with the opposite association at $z=z_{2}$. Note also that for a time-dependent potential energy there 
will be scattering from open-channel input into both open- and closed-channel output, which circumstance is accounted for in Eq. (58). We now define a normalized, Type II wave function as one for which the input amplitude function is normalized to one, that is,

$$
\begin{aligned}
1 & =\iiint_{\text {open }} d^{3} k\left[\left|f_{\text {in }}^{F}(\mathbf{k})\right|^{2}+\left|f_{\text {in }}^{B}(\mathbf{k})\right|^{2}\right] \\
& =\iiint_{\text {open }} d^{3} k\left[\left|f_{\text {out }}^{F}(\mathbf{k})\right|^{2}+\left|f_{\text {out }}^{B}(\mathbf{k})\right|^{2}\right],
\end{aligned}
$$

where the second equation follows from Eqs. (56), (58), and (59). Note that there is no contribution to the output normalization from closed-channel amplitudes.

We conclude the section by developing a formula for the expectation value of the operator $\check{t} I_{2}$ in terms of the wave-number space expansion amplitudes $f^{\zeta}(\mathbf{k})$. Let $\Psi(t, x, y, z)$ be as in Eq. (46). Then we have

$$
\begin{aligned}
\left\langle\check{t} I_{2}\right\rangle_{\Psi(z)}= & \hbar^{-1} \iiint_{\mathbb{R}^{3}} d t d x d y \iiint_{\mathbb{R}^{3}} d^{3} k \sum_{\zeta=B}^{F} \Psi(t, x, y, z)^{\dagger} M \\
& \times f^{\zeta}(\mathbf{k})(2 \pi)^{-3 / 2} t \exp \left[-i k_{t} t+i k_{x} x+i k_{y} y\right] X^{\zeta}(\mathbf{k} ; z) .
\end{aligned}
$$

Replacing $t \exp \left[-i k_{t} t\right]$ by $i\left(\partial / \partial k_{t}\right) \exp \left[-i k_{t} t\right]$ and integrating by parts on $k_{t}$, we find that

$$
\begin{aligned}
\left\langle\check{t} I_{2}\right\rangle_{\Psi(z)}= & \hbar^{-1} \iiint_{\mathbb{R}^{3}} d t d x d y \iiint_{\mathbb{R}^{3}} d^{3} k \sum_{\zeta=B}^{F} \Psi(t, x, y, z)^{\dagger} M \\
& \times(2 \pi)^{-3 / 2} \exp \left[-i k_{t} t+i k_{x} x+i k_{y} y\right] \frac{1}{i} \frac{\partial}{\partial k_{t}}\left[f^{\zeta}(\mathbf{k}) X^{\zeta}(\mathbf{k} ; z)\right] .
\end{aligned}
$$

Analogous to spatial position operators in momentum space, the operator for $t$ transforms into $-i \partial / \partial k_{t}$, the sign difference being a result of the negative sign in the exponent in Eq. (42). This result agrees with that in Ref. 40, Ch. 8, Eq. (286); see also 24, §8. Eq. (62) can be interpreted as yielding a quantummechanical value for the average arrival time, or crossing time, of the particle in the state $\Psi$ at the given $z=$ constant plane.

The evaluation of Eq. (62) is facilitated by the following formulas: for $\zeta=F$ and $B$ and for $\zeta^{\prime}=B$ and $F$, respectively, and for open channels,

$$
\begin{aligned}
\hbar^{-1} X^{\zeta}(\mathbf{k} ; z)^{\dagger} M \frac{1}{i} \frac{\partial X^{\zeta}}{\partial k_{t}}(\mathbf{k} ; z) & =\frac{m z}{\hbar k_{z}} \\
\hbar^{-1} X^{\zeta}(\mathbf{k} ; z)^{\dagger} M \frac{1}{i} \frac{\partial X^{\zeta^{\prime}}}{\partial k_{t}}(\mathbf{k} ; z) & =\sigma(\zeta) \frac{i m}{2 \hbar k_{z}^{2}} \exp \left(-\sigma(\zeta) 2 i k_{z} z\right),
\end{aligned}
$$

while for closed channels

$$
\begin{aligned}
\hbar^{-1} X^{\zeta}(\mathbf{k} ; z)^{\dagger} M \frac{1}{i} \frac{\partial X^{\zeta}}{\partial k_{t}}(\mathbf{k} ; z) & =-\sigma(\zeta) \frac{m}{2 \hbar \kappa_{z}^{2}} \exp \left(-\sigma(\zeta) 2 \kappa_{z} z\right) \\
\hbar^{-1} X^{\zeta}(\mathbf{k} ; z)^{\dagger} M \frac{1}{i} \frac{\partial X^{\zeta^{\prime}}}{\partial k_{t}}(\mathbf{k} ; z) & =\sigma(\zeta) \frac{i m z}{\hbar \kappa_{z}}
\end{aligned}
$$


If we carry out the differentiations in the integrand of Eq. (62), we find that

$$
\begin{aligned}
& \left\langle\check{t} I_{2}\right\rangle_{\Psi(z)}=\iiint_{\text {open }} d^{3} k\left[\sum_{\zeta=B}^{F}\left(\sigma(\zeta) f^{\zeta}(\mathbf{k})^{*} \frac{1}{i} \frac{\partial f^{\zeta}}{\partial k_{t}}(\mathbf{k})\right)\right. \\
& -\frac{i m}{2 \hbar k_{z}^{2}} \exp \left(+2 i k_{z} z\right) f^{B}(\mathbf{k})^{*} f^{F}(\mathbf{k}) \\
& +\frac{i m}{2 \hbar k_{z}^{2}} \exp \left(-2 i k_{z} z\right) f^{F}(\mathbf{k})^{*} f^{B}(\mathbf{k}) \\
& \left.+\frac{m z}{\hbar k_{z}}\left(\left|f^{F}(\mathbf{k})\right|^{2}+\left|f^{B}(\mathbf{k})\right|^{2}\right)\right]+\iiint_{\text {closed }} d^{3} k \\
& \times\left[f^{B}(\mathbf{k})^{*} \frac{1}{i} \frac{\partial f^{F}}{\partial k_{t}}(\mathbf{k})+f^{F}(\mathbf{k})^{*} \frac{1}{i} \frac{\partial f^{B}}{\partial k_{t}}(\mathbf{k})\right. \\
& -\frac{m}{2 \hbar \kappa_{z}^{2}} \exp \left(-2 \kappa_{z} z\right)\left|f^{F}(\mathbf{k})\right|^{2}+\frac{m}{2 \hbar \kappa_{z}^{2}} \exp \left(+2 \kappa_{z} z\right)\left|f^{B}(\mathbf{k})\right|^{2} \\
& \left.-\frac{i m z}{\hbar \kappa_{z}} f^{B}(\mathbf{k})^{*} f^{F}(\mathbf{k})+\frac{i m z}{\hbar \kappa_{z}} f^{F}(\mathbf{k})^{*} f^{B}(\mathbf{k})\right] .
\end{aligned}
$$

Due to the denominators involving $k_{z}^{2}$ or $\kappa_{z}^{2}$ in the above, convergence of the integrals requires that the $f^{\zeta}(\mathbf{k})$ approach zero sufficiently rapidly as $\mathbf{k}$ approaches the boundary between open and closed channels.

\section{Scattering; dwell and delay times}

In this section, we shall presume the presence of a generic potential energy distribution $V(t, x, y, z)$, such that its support is contained within the box $\mathcal{B}_{2}$. The potential energy gives rise to scattering of the (we presume, purely openchannel) input signals, such that reflected and transmitted waves across the spectrum of $\mathbf{k}$, including both open and closed channels, will comprise the output signal from the box. We now assume that our prescription for specifying the input yields necessary and sufficient information such that a solution to the Schrödinger equation within the box exists, satisfies the input boundary conditions, and is unique. Accordingly, the output is determined by the input, and this association must be linear in view of the linearity of the Schrödinger equation. The linear operator specifying this association consists of reflection and transmission coefficients, which can be assembled into an $S$-matrix, which in turn - as we shall verify - has a submatrix, referring to purely open-channel output as well as input, that is unitary.

We presume that the Schrödinger equation has been solved for all openchannel inputs, and express the output linearly in terms of the input as follows: 


$$
\begin{aligned}
f_{\text {out }}^{B}(\mathbf{k}) & =\iiint_{\text {open }} d^{3} k^{\prime} \\
& \times\left[R^{B F}\left(\mathbf{k} ; \mathbf{k}^{\prime}\right) f_{\text {in }}^{F}\left(\mathbf{k}^{\prime}\right)\right. \\
& \left.+T^{B B}\left(\mathbf{k} ; \mathbf{k}^{\prime}\right) f_{\text {in }}^{B}\left(\mathbf{k}^{\prime}\right)\right], \\
f_{\text {out }}^{F}(\mathbf{k}) & =\iiint_{\text {open }} d^{3} k^{\prime} \\
& \times\left[T^{F F}\left(\mathbf{k} ; \mathbf{k}^{\prime}\right) f_{\text {in }}^{F}\left(\mathbf{k}^{\prime}\right)\right. \\
& \left.+R^{F B}\left(\mathbf{k} ; \mathbf{k}^{\prime}\right) f_{\text {in }}^{B}\left(\mathbf{k}^{\prime}\right)\right] .
\end{aligned}
$$

The functions $T^{F F}, R^{F B}, R^{B F}$, and $T^{B B}$ are reflection and transmission coefficients, where the input-to-output superscripts are to be read from right to left. In Eqs. (66) and (67), consistent with Eqs. (58a) and (58b), the reflection and transmission coefficients are defined for the output parameter $k_{t}$ having either an open- or a closed-channel value.

For later convenience, we define

$$
I^{\text {open }}\left(\mathbf{k}^{\prime}-\mathbf{k}^{\prime \prime}\right)=\delta^{\text {open }}\left(k_{t}^{\prime}-k_{t}^{\prime \prime}\right) \delta\left(k_{x}^{\prime}-k_{x}^{\prime \prime}\right) \delta\left(k_{y}^{\prime}-k_{y}^{\prime \prime}\right),
$$

where $\delta^{\text {open }}\left(k_{t}^{\prime}-k_{t}^{\prime \prime}\right)$ is defined only for both $k_{t}^{\prime}$ and $k_{t}^{\prime \prime}$ corresponding to open channels.

Let us now substitute Eqs. (66) and (67) into Eq. (60). We obtain a quadratic expression in the input amplitudes $f_{\text {in }}^{\zeta}$ on both sides of the resulting equation. Since these amplitude functions are arbitrary, the coefficients of the four quadratic terms must be equal. We infer that, for both $k_{t}^{\prime}$ and $k_{t}^{\prime \prime}$ being of open-channel type,

$$
\begin{aligned}
& \iiint_{\text {open }} d^{3} k\left[T^{F F}\left(\mathbf{k} ; \mathbf{k}^{\prime}\right)^{*} T^{F F}\left(\mathbf{k} ; \mathbf{k}^{\prime \prime}\right)\right. \\
& \left.+R^{B F}\left(\mathbf{k} ; \mathbf{k}^{\prime}\right)^{*} R^{B F}\left(\mathbf{k} ; \mathbf{k}^{\prime \prime}\right)\right] \\
& =I^{\mathrm{open}}\left(\mathbf{k}^{\prime}-\mathbf{k}^{\prime \prime}\right), \\
& \iiint_{\mathrm{open}} d^{3} k\left[R^{F B}\left(\mathbf{k} ; \mathbf{k}^{\prime}\right)^{*} R^{F B}\left(\mathbf{k} ; \mathbf{k}^{\prime \prime}\right)\right. \\
& \left.+T^{B B}\left(\mathbf{k} ; \mathbf{k}^{\prime}\right)^{*} T^{B B}\left(\mathbf{k} ; \mathbf{k}^{\prime \prime}\right)\right] \\
& =I^{\mathrm{open}}\left(\mathbf{k}^{\prime}-\mathbf{k}^{\prime \prime}\right), \\
& \iiint_{\mathrm{open}} d^{3} k\left[R^{F B}\left(\mathbf{k} ; \mathbf{k}^{\prime}\right)^{*} T^{F F}\left(\mathbf{k} ; \mathbf{k}^{\prime \prime}\right)\right. \\
& \left.+T^{B B}\left(\mathbf{k} ; \mathbf{k}^{\prime}\right)^{*} R^{B F}\left(\mathbf{k} ; \mathbf{k}^{\prime \prime}\right)\right]=0,
\end{aligned}
$$




$$
\begin{aligned}
& \iiint_{\text {open }} d^{3} k\left[R^{B F}\left(\mathbf{k} ; \mathbf{k}^{\prime}\right)^{*} T^{B B}\left(\mathbf{k} ; \mathbf{k}^{\prime \prime}\right)\right. \\
& \left.+T^{F F}\left(\mathbf{k} ; \mathbf{k}^{\prime}\right)^{*} R^{F B}\left(\mathbf{k} ; \mathbf{k}^{\prime \prime}\right)\right]=0 .
\end{aligned}
$$

Let us now make up an $S$-matrix and its transpose conjugate $S^{\dagger}$ from the reflection and transmission matrices. In the following, the unprimed index $k_{t}$ ranges over all real values, while $k_{t}^{\prime}$ and $k_{t}^{\prime \prime}$ range over open-channel values only:

$$
\begin{aligned}
S\left(\mathbf{k} ; \mathbf{k}^{\prime \prime}\right) & =\left[\begin{array}{ll}
T^{F F}\left(\mathbf{k} ; \mathbf{k}^{\prime \prime}\right) & R^{F B}\left(\mathbf{k} ; \mathbf{k}^{\prime \prime}\right) \\
R^{B F}\left(\mathbf{k} ; \mathbf{k}^{\prime \prime}\right) & T^{B B}\left(\mathbf{k} ; \mathbf{k}^{\prime \prime}\right)
\end{array}\right], \\
S^{\dagger}\left(\mathbf{k}^{\prime} ; \mathbf{k}\right) & =\left[\begin{array}{ll}
T^{F F}\left(\mathbf{k} ; \mathbf{k}^{\prime}\right)^{*} & R^{B F}\left(\mathbf{k} ; \mathbf{k}^{\prime}\right)^{*} \\
R^{F B}\left(\mathbf{k} ; \mathbf{k}^{\prime}\right)^{*} & T^{B B}\left(\mathbf{k} ; \mathbf{k}^{\prime}\right)^{*}
\end{array}\right] .
\end{aligned}
$$

It is convenient to define two submatrices of $S$, the open-channel part $S_{o}$ and the closed-channel part $S_{c}$ as follows:

$$
\begin{aligned}
& S_{o}\left(\mathbf{k} ; \mathbf{k}^{\prime}\right)=S\left(\mathbf{k} ; \mathbf{k}^{\prime}\right) \text {, for all } k_{t}>(\hbar / 2 m)\left(k_{x}^{2}+k_{y}^{2}\right) \text {, } \\
& S_{c}\left(\mathbf{k} ; \mathbf{k}^{\prime}\right)=S\left(\mathbf{k} ; \mathbf{k}^{\prime}\right) \text {, for all } k_{t}<(\hbar / 2 m)\left(k_{x}^{2}+k_{y}^{2}\right) \text {. }
\end{aligned}
$$

Then $S_{o}$ is unitary on the left as a result of Eqs. (69)-(72):

$$
\begin{aligned}
\left(S_{o}^{\dagger} S_{o}\right)\left(\mathbf{k}^{\prime} ; \mathbf{k}^{\prime \prime}\right) & =\iiint_{\text {open }} d^{3} k\left[S_{o}^{\dagger}\left(\mathbf{k}^{\prime} ; \mathbf{k}\right) S_{o}\left(\mathbf{k} ; \mathbf{k}^{\prime \prime}\right)\right] \\
& =I_{2} \otimes I^{\text {open }}\left(\mathbf{k}^{\prime}-\mathbf{k}^{\prime \prime}\right) .
\end{aligned}
$$

One expects that $S_{o}$ is also unitary on the right,

$$
\left(S_{o} S_{o}^{\dagger}\right)\left(\mathbf{k}^{\prime} ; \mathbf{k}^{\prime \prime}\right)=I_{2} \otimes I^{\mathrm{open}}\left(\mathbf{k}^{\prime}-\mathbf{k}^{\prime \prime}\right) .
$$

We now reduce the formulas for the expectation values of the operator $\check{t} I_{2}$ at $z=z_{1}, z_{2}$, using Eqs. (58), 65], 666), and (67), and then establish a relatively simple form for the difference of the two values. We assume that the input amplitudes $f_{\text {in }}^{\zeta}(\mathbf{k})$ and the output state values of the $S$-matrix elements go to zero at the open/closed-channel threshold so that the following integrals converge. We have first

$$
\begin{aligned}
\left\langle\check{t} I_{2}\right\rangle_{\Psi\left(z_{1}\right)} & =\iiint_{\text {open }} d^{3} k f_{\text {in }}^{F}(\mathbf{k})^{*} \frac{1}{i} \frac{\partial f_{\text {in }}^{F}}{\partial k_{t}}(\mathbf{k}) \\
& +\iiint_{\text {open }} d^{3} k \iiint_{\text {open }} d^{3} k^{\prime} \sum_{\zeta, \zeta^{\prime}} \\
& \times f_{\text {in }}^{\zeta}(\mathbf{k})^{*} A^{\zeta \zeta^{\prime}}\left(\mathbf{k} ; \mathbf{k}^{\prime} ; z_{1}\right) f_{\text {in }}^{\zeta^{\prime}}\left(\mathbf{k}^{\prime}\right) .
\end{aligned}
$$


The matrix of coefficients is as follows:

$$
\begin{aligned}
& A^{F F}\left(\mathbf{k} ; \mathbf{k}^{\prime} ; z_{1}\right) \\
& =\frac{m z_{1}}{\hbar k_{z}} I^{\text {open }}\left(\mathbf{k}-\mathbf{k}^{\prime}\right) \\
& +\frac{i m}{2 \hbar k_{z}^{2}} \exp \left(-2 i k_{z} z_{1}\right) R^{B F}\left(\mathbf{k} ; \mathbf{k}^{\prime}\right) \\
& -R^{B F}\left(\mathbf{k}^{\prime} ; \mathbf{k}\right)^{*} \frac{i m}{2 \hbar k_{z}^{\prime 2}} \exp \left(+2 i k_{z}^{\prime} z_{1}\right) \\
& +\iiint_{\text {open }} d^{3} k^{\prime \prime} R^{B F}\left(\mathbf{k}^{\prime \prime} ; \mathbf{k}\right)^{*} \\
& \times\left[\frac{m z_{1}}{\hbar k_{z}^{\prime \prime}}-\frac{1}{i} \frac{\partial}{\partial k_{t}^{\prime \prime}}\right] R^{B F}\left(\mathbf{k}^{\prime \prime} ; \mathbf{k}^{\prime}\right) \\
& +\iiint_{\text {closed }} d^{3} k^{\prime \prime} R^{B F}\left(\mathbf{k}^{\prime \prime} ; \mathbf{k}\right)^{*} \\
& \times \frac{m}{2 \hbar \kappa_{z}^{\prime \prime 2}} \exp \left(2 \kappa_{z}^{\prime \prime} z_{1}\right) R^{B F}\left(\mathbf{k}^{\prime \prime} ; \mathbf{k}^{\prime}\right) \\
& A^{F B}\left(\mathbf{k} ; \mathbf{k}^{\prime} ; z_{1}\right) \\
& =\frac{i m}{2 \hbar k_{z}^{2}} \exp \left(-2 i k_{z} z_{1}\right) T^{B B}\left(\mathbf{k} ; \mathbf{k}^{\prime}\right) \\
& +\iiint_{\text {open }} d^{3} k^{\prime \prime} R^{B F}\left(\mathbf{k}^{\prime \prime} ; \mathbf{k}\right)^{*} \\
& \times\left[\frac{m z_{1}}{\hbar k_{z}^{\prime \prime}}-\frac{1}{i} \frac{\partial}{\partial k_{t}^{\prime \prime}}\right] T^{B B}\left(\mathbf{k}^{\prime \prime} ; \mathbf{k}^{\prime}\right) \\
& +\iiint_{\text {closed }} d^{3} k^{\prime \prime} R^{B F}\left(\mathbf{k}^{\prime \prime} ; \mathbf{k}\right)^{*} \\
& \times \frac{m}{2 \hbar \kappa_{z}^{\prime \prime 2}} \exp \left(2 \kappa_{z}^{\prime \prime} z_{1}\right) T^{B B}\left(\mathbf{k}^{\prime \prime} ; \mathbf{k}^{\prime}\right) \\
& A^{B F}\left(\mathbf{k} ; \mathbf{k}^{\prime} ; z_{1}\right) \\
& =-T^{B B}\left(\mathbf{k}^{\prime} ; \mathbf{k}\right)^{*} \frac{i m}{2 \hbar k_{z}^{\prime 2}} \exp \left(2 i k_{z}^{\prime} z_{1}\right) \\
& +\iiint_{\text {open }} d^{3} k^{\prime \prime} T^{B B}\left(\mathbf{k}^{\prime \prime} ; \mathbf{k}\right)^{*} \\
& \times\left[\frac{m z_{1}}{\hbar k_{z}^{\prime \prime}}-\frac{1}{i} \frac{\partial}{\partial k_{t}^{\prime \prime}}\right] R^{B F}\left(\mathbf{k}^{\prime \prime} ; \mathbf{k}^{\prime}\right) \\
& +\iiint_{\text {closed }} d^{3} k^{\prime \prime} T^{B B}\left(\mathbf{k}^{\prime \prime} ; \mathbf{k}\right)^{*} \\
& \times \frac{m}{2 \hbar \kappa_{z}^{\prime \prime 2}} \exp \left(2 \kappa_{z}^{\prime \prime} z_{1}\right) R^{B F}\left(\mathbf{k}^{\prime \prime} ; \mathbf{k}^{\prime}\right) \text {; }
\end{aligned}
$$




$$
\begin{aligned}
A^{B B}\left(\mathbf{k} ; \mathbf{k}^{\prime} ; z_{1}\right) & \\
& =\iiint_{\text {open }} d^{3} k^{\prime \prime} T^{B B}\left(\mathbf{k}^{\prime \prime} ; \mathbf{k}\right)^{*} \\
& \times\left[\frac{m z_{1}}{\hbar k_{z}^{\prime \prime}}-\frac{1}{i} \frac{\partial}{\partial k_{t}^{\prime \prime}}\right] T^{B B}\left(\mathbf{k}^{\prime \prime} ; \mathbf{k}^{\prime}\right) \\
& +\iiint_{\text {closed }} d^{3} k^{\prime \prime} T^{B B}\left(\mathbf{k}^{\prime \prime} ; \mathbf{k}\right)^{*} \\
& \times \frac{m}{2 \hbar \kappa_{z}^{\prime \prime 2}} \exp \left(2 \kappa_{z}^{\prime \prime} z_{1}\right) T^{B B}\left(\mathbf{k}^{\prime \prime} ; \mathbf{k}^{\prime}\right) .
\end{aligned}
$$

At $z=z_{2}$ we have

$$
\begin{aligned}
\left\langle\check{t} I_{2}\right\rangle_{\Psi\left(z_{2}\right)} & =-\iiint_{\text {open }} d^{3} k f_{\text {in }}^{B}(\mathbf{k})^{*} \frac{1}{i} \frac{\partial f_{\text {in }}^{B}}{\partial k_{t}}(\mathbf{k}) \\
& +\iint_{\text {open }} d^{3} k \iiint_{\text {open }} d^{3} k^{\prime} \sum_{\zeta, \zeta^{\prime}} \\
& \times f_{\text {in }}^{\zeta}(\mathbf{k})^{*} C^{\zeta \zeta^{\prime}}\left(\mathbf{k} ; \mathbf{k}^{\prime} ; z_{2}\right) f_{\mathrm{in}}^{\zeta^{\prime}}\left(\mathbf{k}^{\prime}\right) .
\end{aligned}
$$

In the above, the coefficient matrices are

$$
\begin{aligned}
& C^{F F}\left(\mathbf{k} ; \mathbf{k}^{\prime} ; z_{2}\right) \\
&= \iiint_{\text {open }} d^{3} k^{\prime \prime} T^{F F}\left(\mathbf{k}^{\prime \prime} ; \mathbf{k}\right)^{*} \\
& \times\left[\frac{m z_{2}}{\hbar k_{z}^{\prime \prime}}+\frac{1}{i} \frac{\partial}{\partial k_{t}^{\prime \prime}}\right] T^{F F}\left(\mathbf{k}^{\prime \prime} ; \mathbf{k}^{\prime}\right) \\
&-\iiint_{\text {closed }} d^{3} k^{\prime \prime} T^{F F}\left(\mathbf{k}^{\prime \prime} ; \mathbf{k}\right)^{*} \\
& \times \frac{m}{2 \hbar \kappa_{z}^{\prime \prime 2}} \exp \left(-2 \kappa_{z}^{\prime \prime} z_{2}\right) T^{F F}\left(\mathbf{k}^{\prime \prime} ; \mathbf{k}^{\prime}\right) ; \\
& C^{F B}\left(\mathbf{k} ; \mathbf{k}^{\prime} ; z_{2}\right) \\
&=\quad T^{F F}\left(\mathbf{k}^{\prime} ; \mathbf{k}\right)^{*} \frac{i m}{2 \hbar k_{z}^{\prime 2}} \exp \left(-2 i k_{z}^{\prime} z_{2}\right) \\
& \quad+\iiint_{\text {open }} d^{3} k^{\prime \prime} T^{F F}\left(\mathbf{k}^{\prime \prime} ; \mathbf{k}\right)^{*} \\
& \quad \times\left[\frac{m z_{2}}{\hbar k_{z}^{\prime \prime}}+\frac{1}{i} \frac{\partial}{\partial k_{t}^{\prime \prime}}\right] R^{F B}\left(\mathbf{k}^{\prime \prime} ; \mathbf{k}\right) \\
&-\iiint_{\text {closed }} d^{3} k^{\prime \prime} T^{F F}\left(\mathbf{k}^{\prime \prime} ; \mathbf{k}\right)^{*} \\
& \quad \times \frac{m}{2 \hbar \kappa_{z}^{\prime \prime 2}} \exp \left(-2 \kappa_{z}^{\prime \prime} z_{2}\right) R^{F B}\left(\mathbf{k}^{\prime \prime} ; \mathbf{k}^{\prime}\right) ;
\end{aligned}
$$




$$
\begin{aligned}
& C^{B F}\left(\mathbf{k} ; \mathbf{k}^{\prime} ; z_{2}\right) \\
&=-\frac{i m}{2 \hbar k_{z}^{2}} \exp \left(2 i k_{z} z_{2}\right) T^{F F}\left(\mathbf{k} ; \mathbf{k}^{\prime}\right) \\
&+\iiint_{\text {open }} d^{3} k^{\prime \prime} R^{F B}\left(\mathbf{k}^{\prime \prime} ; \mathbf{k}\right)^{*} \\
& \times\left[\frac{m z_{2}}{\hbar k_{z}^{\prime \prime}}+\frac{1}{i} \frac{\partial}{\partial k_{t}^{\prime \prime}}\right] T^{F F}\left(\mathbf{k}^{\prime \prime} ; \mathbf{k}^{\prime}\right) \\
&-\iiint_{\text {closed }} d^{3} k^{\prime \prime} R^{F B}\left(\mathbf{k}^{\prime \prime} ; \mathbf{k}\right)^{*} \\
& \times \frac{m}{2 \hbar \kappa_{z}^{\prime \prime 2}} \exp \left(-2 \kappa_{z}^{\prime \prime} z_{2}\right) T^{F F}\left(\mathbf{k}^{\prime \prime} ; \mathbf{k}^{\prime}\right) ; \\
& C^{B B}\left(\mathbf{k} ; \mathbf{k}^{\prime} ; z_{2}\right) \\
&= \frac{m z_{2}}{\hbar k_{z}} I^{\text {open }}\left(\mathbf{k}-\mathbf{k}^{\prime}\right) \\
&-\frac{i m}{2 \hbar k_{z}^{2}} \exp \left(2 i k_{z} z_{2}\right) R^{F B}\left(\mathbf{k} ; \mathbf{k}^{\prime}\right) \\
&+R^{F B}\left(\mathbf{k}^{\prime} ; \mathbf{k}\right)^{*} \frac{i m}{2 \hbar k_{z}^{\prime}} \exp \left(-2 i k_{z}^{\prime} z_{2}\right) \\
&+\iiint_{\text {open }} d^{3} k^{\prime \prime} R^{F B}\left(\mathbf{k}^{\prime \prime} ; \mathbf{k}\right)^{*} \\
& \times\left[\frac{m z_{2}}{\hbar k_{z}^{\prime \prime}}+\frac{1}{i} \frac{\partial}{\partial k_{t}^{\prime \prime}}\right] R^{F B}\left(\mathbf{k}^{\prime \prime} ; \mathbf{k}^{\prime}\right) \\
&-\iiint_{\operatorname{closed}} d^{3} k^{\prime \prime} R^{F B}\left(\mathbf{k}^{\prime \prime} ; \mathbf{k}^{\prime}\right)^{*} \\
& \times \frac{m}{2 \hbar \kappa_{z}^{\prime \prime 2}} \exp \left(-2 \kappa_{z}^{\prime \prime} z_{2}\right) R^{F B}\left(\mathbf{k}^{\prime \prime} ; \mathbf{k}^{\prime}\right) .
\end{aligned}
$$

The difference of the two expectation values takes the form

$$
\begin{array}{r}
\left\langle\check{t} I_{2}\right\rangle_{\Psi\left(z_{2}\right)}-\left\langle\check{t} I_{2}\right\rangle_{\Psi\left(z_{1}\right)}=\iiint_{\text {open }} d^{3} k \iiint_{\text {open }} d^{3} k^{\prime} \\
\times \sum_{\zeta, \zeta^{\prime}} f_{\text {in }}^{\zeta}(\mathbf{k})^{*} D^{\zeta \zeta^{\prime}}\left(\mathbf{k} ; \mathbf{k}^{\prime} ; z_{1} ; z_{2}\right) f_{\text {in }}^{\zeta^{\prime}}\left(\mathbf{k}^{\prime}\right) .
\end{array}
$$

We break the $D$-matrices into constituents:

$$
D=D_{1}+M D_{2} S_{o}+S_{o}^{\dagger} D_{2}^{\dagger} M+S_{o}^{\dagger} D_{3} S_{o}+S_{c}^{\dagger} D_{4} S_{c},
$$

where

$$
D_{1}=I^{\mathrm{open}}\left(\mathbf{k}-\mathbf{k}^{\prime}\right) \otimes \operatorname{diag}\left(-\frac{1}{i} \frac{\partial}{\partial k_{t}^{\prime}}-\frac{m z_{1}}{\hbar k_{z}^{\prime}},-\frac{1}{i} \frac{\partial}{\partial k_{t}^{\prime}}+\frac{m z_{2}}{\hbar k_{z}^{\prime}}\right),
$$




$$
\begin{gathered}
D_{2}=\operatorname{diag}\left(\frac{m}{2 \hbar k_{z}^{2}} \exp \left(2 i k_{z} z_{2}\right),-\frac{m}{2 \hbar k_{z}^{2}} \exp \left(-2 i k_{z} z_{1}\right)\right) \\
D_{3}=\operatorname{diag}\left(\frac{1}{i} \frac{\partial}{\partial k_{t}^{\prime \prime}}+\frac{m z_{2}}{\hbar k_{z}^{\prime \prime}}, \frac{1}{i} \frac{\partial}{\partial k_{t}^{\prime \prime}}-\frac{m z_{1}}{\hbar k_{z}^{\prime \prime}}\right) \\
D_{4}=\operatorname{diag}\left(-\frac{m}{2 \hbar \kappa_{z}^{\prime \prime 2}} \exp \left(-2 \kappa_{z}^{\prime \prime} z_{2}\right),-\frac{m}{2 \hbar \kappa_{z}^{\prime \prime 2}} \exp \left(2 \kappa_{z}^{\prime \prime} z_{1}\right)\right) .
\end{gathered}
$$

In Eqs. (92) and (93), the double primes indicate the dummy variables of integration implicit in the final two summands on the rhs of Eq. (89). The $D_{1}$ and $D_{3}$ terms were obtained by Smith [41, 42 for the case that the evolution coordinate is radial and the interaction is time-independent, but otherwise general. Note that Smith's $S$-matrix is the transpose of the $S$-matrix defined above.

It is plausible that Eq. [88, given that the total input is normalized as in Eq. (60), provides a complete expression for the mean dwell time of the particle in the box, inasmuch as it is also an expression for the space-time integral over the box of the divergence of the flow vector density of time. We remark that if the potential energy is time-independent, then the $S_{o}$-matrix takes the form $S_{o}\left(\mathbf{k}^{\prime \prime} ; \mathbf{k}^{\prime}\right)=\delta^{\text {open }}\left(k_{t}^{\prime \prime}-k_{t}^{\prime}\right) \bar{S}_{o}\left(k_{t}^{\prime \prime}, k_{x}^{\prime \prime}, k_{y}^{\prime \prime} ; k_{x}^{\prime}, k_{y}^{\prime}\right)$; one can now show that, due to the unitarity of $S_{o}$, the terms involving $-i \partial f_{\text {in }}^{\zeta} / \partial k_{t}^{\prime}\left(\mathbf{k}^{\prime}\right)$ cancel out in the overall expression for the dwell time. This cancellation does not, as we shall see, occur for the individual delay times for transmission or reflection from a zone of time-independent interaction.

The average delay times that are measured in beam experiments for either transmission or reflection are not so fundamentally defined. We simplify the problem as follows: First, we assume that only one kind of input, that is $\mathrm{F}$ or but not and B, is present. Second, we assume that the closed-channel contributions will be negligible in the measuring apparatus. Third, we neglect interference between the incoming signal and the outgoing signal in the case of reflection (hence, the contributions linear in the $S$-matrix are discarded). We now specify what remains after these simplifications.

In the first instance, let $f_{\text {in }}^{B}(\mathbf{k}) \equiv 0$, and let $f_{\text {in }}^{F}$ be normalized as in Eq. (60). The net outgoing reflected and transmitted currents of particle presence are called $\mathcal{R}_{B \leftarrow F}^{\text {out }}\left(z_{1}\right)$ and $\mathcal{T}_{F \leftarrow F}^{\text {out }}\left(z_{2}\right)$, respectively, and take the values

$$
\begin{aligned}
\mathcal{R}_{B \leftarrow F}^{\text {out }}\left(z_{1}\right)= & \iiint_{\text {open }} d^{3} k \iiint_{\text {open }} d^{3} k^{\prime} \iiint_{\text {open }} d^{3} k^{\prime \prime}\left[f_{\text {in }}^{F}(\mathbf{k})^{*}\right. \\
& \left.\times(-1) R^{B F}\left(\mathbf{k}^{\prime \prime} ; \mathbf{k}\right)^{*} R^{B F}\left(\mathbf{k}^{\prime \prime} ; \mathbf{k}^{\prime}\right) f_{\text {in }}^{F}\left(\mathbf{k}^{\prime}\right)\right], \\
\mathcal{T}_{F \leftarrow F}^{\text {out }}\left(z_{2}\right)= & \iiint_{\text {open }} d^{3} k \iiint_{\text {open }} d^{3} k^{\prime} \iiint_{\text {open }} d^{3} k^{\prime \prime}\left[f_{\text {in }}^{F}(\mathbf{k})^{*}\right. \\
& \left.\times T^{F F}\left(\mathbf{k}^{\prime \prime} ; \mathbf{k}\right)^{*} T^{F F}\left(\mathbf{k}^{\prime \prime} ; \mathbf{k}^{\prime}\right) f_{\text {in }}^{F}\left(\mathbf{k}^{\prime}\right)\right] .
\end{aligned}
$$

According to Eq. (69), we have

$$
\mathcal{T}_{F \leftarrow F}^{\text {out }}\left(z_{2}\right)-\mathcal{R}_{B \leftarrow F}^{\text {out }}\left(z_{1}\right)=1 .
$$


The mean currents of time at entry and upon reflection at $z_{1}$, and upon transmission at $z_{2}$, will be called, respectively, $\tau_{F}^{\text {in }}\left(z_{1}\right), \tau_{B \leftarrow F}^{\text {out }}\left(z_{1}\right)$, and $\tau_{F \leftarrow F}^{\text {out }}\left(z_{2}\right)$, and can be inferred from Eqs. (79) (twice) and (84), subject to the three simplifications spelled out in the previous paragraph, as follows:

$$
\begin{aligned}
\tau_{F}^{\text {in }}\left(z_{1}\right) & =\iiint_{\text {open }} d^{3} k f_{\text {in }}^{F}(\mathbf{k})^{*}\left[\frac{1}{i} \frac{\partial}{\partial k_{t}}+\frac{m z_{1}}{\hbar k_{z}}\right] f_{\text {in }}^{F}(\mathbf{k}) \\
\tau_{B \leftarrow F}^{\text {out }}\left(z_{1}\right) & =\iiint_{\text {open }} d^{3} k \iiint_{\text {open }} d^{3} k^{\prime}\left\{f_{\text {in }}^{F}(\mathbf{k})^{*} \iiint_{\text {open }} d^{3} k^{\prime \prime} R^{B F}\left(\mathbf{k}^{\prime \prime} ; \mathbf{k}\right)^{*}\right. \\
& \left.\left.\times\left[-\frac{1}{i} \frac{\partial}{\partial k_{t}^{\prime \prime}}+\frac{m z_{1}}{\hbar k_{z}^{\prime \prime}}\right] R^{B F}\left(\mathbf{k}^{\prime \prime} ; \mathbf{k}^{\prime}\right)\right] f_{\text {in }}^{F}\left(\mathbf{k}^{\prime}\right)\right\} \\
\tau_{F \leftarrow F}^{\text {out }}\left(z_{2}\right) & =\iiint_{\text {open }} d^{3} k \iiint_{\text {open }} d^{3} k^{\prime}\left\{f_{\text {in }}^{F}(\mathbf{k})^{*} \iiint_{\text {open }} d^{3} k^{\prime \prime} T^{F F}\left(\mathbf{k}^{\prime \prime} ; \mathbf{k}\right)^{*}\right. \\
& \left.\left.\times\left[\frac{1}{i} \frac{\partial}{\partial k_{t}^{\prime \prime}}+\frac{m z_{2}}{\hbar k_{z}^{\prime \prime}}\right] T^{F F}\left(\mathbf{k}^{\prime \prime} ; \mathbf{k}^{\prime}\right)\right] f_{\text {in }}^{F}\left(\mathbf{k}^{\prime}\right)\right\} .
\end{aligned}
$$

In the second instance, let $f_{\text {in }}^{F}(\mathbf{k}) \equiv 0$, and let $f_{\text {in }}^{B}(\mathbf{k})$ be normalized as in Eq. (60). The net outgoing reflected and transmitted currents of particle presence are called $\mathcal{R}_{F \leftarrow B}^{\text {out }}\left(z_{2}\right)$ and $\mathcal{T}_{B \leftarrow B}^{\text {out }}\left(z_{1}\right)$, respectively, and take the values

$$
\begin{aligned}
\mathcal{R}_{F \leftarrow B}^{\text {out }}\left(z_{2}\right)= & \iiint_{\text {open }} d^{3} k \iiint_{\text {open }} d^{3} k^{\prime} \iiint_{\text {open }} d^{3} k^{\prime \prime}\left[f_{\text {in }}^{B}(\mathbf{k})^{*}\right. \\
& \left.\times R^{F B}\left(\mathbf{k}^{\prime \prime} ; \mathbf{k}\right)^{*} R^{F B}\left(\mathbf{k}^{\prime \prime} ; \mathbf{k}^{\prime}\right) f_{\text {in }}^{B}\left(\mathbf{k}^{\prime}\right)\right] \\
\mathcal{T}_{B \leftarrow B}^{\text {out }}\left(z_{1}\right)= & \iiint_{\text {open }} d^{3} k \iiint_{\text {open }} d^{3} k^{\prime} \iiint_{\text {open }} d^{3} k^{\prime \prime}\left[f_{\text {in }}^{B}(\mathbf{k})^{*} T^{B B}\left(\mathbf{k}^{\prime \prime} ; \mathbf{k}\right)^{*}\right. \\
& \left.\times(-1) T^{B B}\left(\mathbf{k}^{\prime \prime} ; \mathbf{k}^{\prime}\right) f_{\text {in }}^{B}\left(\mathbf{k}^{\prime}\right)\right] .
\end{aligned}
$$

According to Eq. (70), we have

$$
\mathcal{R}_{F \leftarrow B}^{\text {out }}\left(z_{2}\right)-\mathcal{T}_{B \leftarrow B}^{\text {out }}\left(z_{1}\right)=1 .
$$

The net currents of time at $z_{2}$ upon entry and after reflection, and at $z_{1}$ after transmission, will be called, respectively, $\tau_{B}^{\text {in }}\left(z_{2}\right), \tau_{F \leftarrow B}^{\text {out }}\left(z_{2}\right)$, and $\tau_{B \leftarrow B}^{\text {out }}\left(z_{1}\right)$, and can be inferred from Eqs. (87) (twice) and (82), subject to the three simplifica- 
tions given previously, as follows:

$$
\begin{aligned}
\tau_{B}^{\text {in }}\left(z_{2}\right) & =\iiint_{\text {open }} d^{3} k f_{\text {in }}^{B}(\mathbf{k})^{*}\left[-\frac{1}{i} \frac{\partial}{\partial k_{t}}+\frac{m z_{2}}{\hbar k_{z}}\right] f_{\text {in }}^{B}(\mathbf{k}), \\
\tau_{F \leftarrow B}^{\text {out }}\left(z_{2}\right) & =\iiint_{\text {open }} d^{3} k \iiint_{\text {open }} d^{3} k^{\prime}\left\{f_{\text {in }}^{B}(\mathbf{k})^{*} \iiint_{\text {open }} d^{3} k^{\prime \prime} R^{F B}\left(\mathbf{k}^{\prime \prime} ; \mathbf{k}\right)^{*}\right. \\
& \left.\left.\times\left[\frac{1}{i} \frac{\partial}{\partial k_{t}^{\prime \prime}}+\frac{m z_{2}}{\hbar k_{z}^{\prime \prime}}\right] R^{F B}\left(\mathbf{k}^{\prime \prime} ; \mathbf{k}^{\prime}\right)\right] f_{\text {in }}^{B}\left(\mathbf{k}^{\prime}\right)\right\}, \\
\tau_{B \leftarrow B}^{\text {out }}\left(z_{1}\right) & =\iiint_{\text {open }} d^{3} k \iiint_{\text {open }} d^{3} k^{\prime}\left\{f_{\text {in }}^{B}(\mathbf{k})^{*} \iiint_{\text {open }} d^{3} k^{\prime \prime} T^{B B}\left(\mathbf{k}^{\prime \prime} ; \mathbf{k}\right)^{*}\right. \\
& \left.\left.\times\left[-\frac{1}{i} \frac{\partial}{\partial k_{t}^{\prime \prime}}+\frac{m z_{1}}{\hbar k_{z}^{\prime \prime}}\right] T^{B B}\left(\mathbf{k}^{\prime \prime} ; \mathbf{k}^{\prime}\right)\right] f_{\text {in }}^{B}\left(\mathbf{k}^{\prime}\right)\right\} .
\end{aligned}
$$

We now undertake to use the derived results to obtain estimates for the average delay time for the four processes of transmission and reflection. Due to the absence of space- and time-reversal symmetry of the potential energy, there will be no special relationships between the two transmission times or between the two reflection times. Let the transmission delay times be called $\tau_{F \leftarrow F}^{\text {trans }}\left(z_{2} \leftarrow z_{1}\right)$ and $\tau_{B \leftarrow B}^{\text {trans }}\left(z_{1} \leftarrow z_{2}\right)$, while the reflection delay times are called $\tau_{B \leftarrow F}^{\text {reff }}\left(z_{1} \leftarrow z_{1}\right)$ and $\tau_{F \leftarrow B}^{\text {reff }}\left(z_{2} \leftarrow z_{2}\right)$. We proceed from the following principle for computing delay times (currents are taken with their algebraic signs intact):

$$
\begin{aligned}
\text { delay time }= & \frac{\text { (output current of time across exit plane) }}{\text { (output particle current across exit plane) }} \\
& -\frac{\text { (input current of time across entry plane) }}{\text { (input particle current across entry plane) }},
\end{aligned}
$$

where the exit plane is the same, or the opposite, as the entry plane on reflection, or on transmission, respectively. (Similar formulas appear in [3], p. 110, Eqs. (26) and (27), in [15, Eqs. (28) and (29), in [44, Eqs. (16) and (17), in [45], Eq. (61), and in [46, Eq. (1).) We therefore have that

$$
\begin{aligned}
& \tau_{F \leftarrow F}^{\text {trans }}\left(z_{2} \leftarrow z_{1}\right)=\frac{\tau_{F \leftarrow F}^{\text {out }}\left(z_{2}\right)}{\mathcal{T}_{F \leftarrow F}^{\text {out }}\left(z_{2}\right)}-\tau_{F}^{\text {in }}\left(z_{1}\right), \\
& \tau_{B \leftarrow F}^{\text {reff }}\left(z_{1} \leftarrow z_{1}\right)=\frac{\tau_{B \leftarrow F}^{\text {out }}\left(z_{1}\right)}{\mathcal{R}_{B \leftarrow F}^{\text {out }}\left(z_{1}\right)}-\tau_{F}^{\text {in }}\left(z_{1}\right), \\
& \tau_{B \leftarrow B}^{\text {trans }}\left(z_{1} \leftarrow z_{2}\right)=\frac{\tau_{B \leftarrow B}^{\text {out }}\left(z_{1}\right)}{\mathcal{T}_{B \leftarrow B}^{\text {out }}\left(z_{1}\right)}+\tau_{B}^{\text {in }}\left(z_{2}\right), \\
& \tau_{F \leftarrow B}^{\text {ref }}\left(z_{2} \leftarrow z_{2}\right)=\frac{\tau_{F \leftarrow B}^{\text {out }}\left(z_{2}\right)}{\mathcal{R}_{F \leftarrow B}^{\text {out }}\left(z_{2}\right)}+\tau_{B}^{\text {in }}\left(z_{2}\right) .
\end{aligned}
$$

\section{Discussion}

We have shown that the time can be construed as an observable in the context of the Schrödinger equation. We shall now undertake the discussion of some 
aspects of the physical interpretation of the formalism, and of the relation of the present work to certain other similar investigations.

For a state $\Psi(t, x, y, z)$ made up of a finite number of closed-channel amplitudes that are all exponentially decreasing with $z$ increasing, the net current of particle presence crossing any $z=$ constant surface is zero - see Eq. (56). Zero net current means that the particle will, in a large number of experiments, cross negatively as often as it crosses positively. The exponential decrease of the wave function is consistent with a picture of a "virtual" particle being created in the interaction region, propagating a small distance positively, and then falling back into the region where it was created. For $F$-type closed-channel states we say that the state propagates in the positive $z$ direction, but represents an equal flux of particle motion in positive and negative $z$-directions. For open-channel $F$-(B-)type states, propagation and global particle motion are jointly positive (negative).

It is possible, with the given physical system, to make a measurement of local (in $(t, x, y)$ ) net transit flux of particles across a proper subset of the $z=$ constant plane. A local measurement of either the positive or the negative direction in $z$ of a particle's transit involves noncommuting projection operators, one in position space and one in wavenumber space. That is, we would then ask two incompatible yes/no questions: (1) Did the particle cross the plane in a given proper $(t, x, y)$ subregion? (2) Did the particle cross positively/negatively in the $z$-direction? The outcome depends on the details of an often-repeated measurement on the system with the same input in each trial. To be sure, taking a sufficiently large subset of the $z=$ constant plane for asking question (1) will, to a good approximation, be close to taking all of it, so that question (2) can be answered with negligible inconsistency. Also, the question "What is the difference between the numbers of particles crossing positively and particles crossing negatively across a small subregion of the $z$-plane?" involves no inconsistency, and yields a result predictable from the local wave function alone; it is the separate local densities of positive and of negative crossings that depend on the measurement scheme.

A substantial effort has been dedicated to the establishment of a time-energy uncertainty principle - see the discussion and references in [47. An uncertainty principle appears to be associated with a positive definite metric, a requirement that we have dropped. It is not obviously impossible to formulate some kind of analogous principle involving the time and its conjugate momentum $p_{t}$ within the present formalism in special circumstances, but the author has not been successful in finding such circumstances.

The Schrödinger equation can create spatially and temporally localized eddies of probability current: even though a wave is made up entirely of a packet of $F$-type open-channel states, this current density can be negative in a neighborhood (see the examples by Kijowski and Mielnik cited in the following paragraph) and therefore will be greater than one in a complementary set of the given $z=$ constant plane. Hence, even for a packet of only free-particle $F$-type states this normalized particle count can fall outside the interval $[0,1]$ on proper subsets of a $z=$ constant plane, and is not a probability, although a transition 
process, as in the conventional interpretation of quantum mechanics, has irreducible randomness and is unpredictable in detail in contrast to a classical process. A further complication results from the circumstance that this normalized particle count is not, when closed channels are present, even globally (i.e., across an entire $z=$ constant plane) an algebraic sum of two separate currents due to forward- and backward-propagating particles, as there can be nonzero global interference between the $F$ - and $B$-type closed-channel contributions to the total current.

Kijowski [24, 48] undertook to establish a time-energy uncertainty principle by analyzing the evolution of a Schrödinger wave function in a space-like direction, and in this respect there is overlap between Kijowski's work and the present undertaking. Kijowski's first "unsuccessful attempt" (24, §3) begins in a similar manner to that proposed above, but his inner product law does not involve an integral over time; since, as noted following Eq. (50), the wave functions of Eq. (49) do not satisfy the Cauchy inequality, the interference terms in a local inner product can make the current density negative for the superposition of two forward-propagating open channel states, as shown in an example in [24], 33 . Mielnik (26], $§ 5$, Lemma) noted that a Schrödinger wave packet that at $t=0$ has its source entirely to the left of $z=0$, say, could eventually give rise to probability currents normal to the $z=0$ plane that need not be everywhere positive. Similarly, the integrand for the particle current for the norm of a superposition of $F$-type open-channel states in Eq. (28) need not be everywhere nonnegative. These local negative currents all result from interference terms that yield zero net contribution in the present formalism due to the integral over $t, x$ and $y$ in the inner product.

Kijowski's formalism is substantially different from the present one - the norm of an $F$-type state is given in 24], Eq. (9) - but in which the average time of crossing a spatial wall for $F$-type states nevertheless reduces to the same form (24, $§ 10)$ in terms of the probability current as Eq. (37). There are discussions of Kijowski's work in [49, §1.5.1, and [50], $§ 10.2$.

Mielnik 26] critiques both Kijowski's 24] and Piron's 25] attempts to establish formalisms for spacewise propagation of a wave function, concludes that they do not offer a solution to the problem of defining time as an observable, and makes no additional proposals along these lines. Although the initial ideas of the two latter papers resemble that of the present paper, the respective implementations differ considerably, so we shall not attempt further review of them here.

Another question concerns the generalization of the effect of a measurement on a wave function that propagates in both directions away from the surface on which the measurement is performed. Suppose in fact that, in a problem of type II, two adjacent boxes occupy the space-like intervals $\left[z_{1}, z_{2}\right]$ and $\left[z_{2}, z_{3}\right]$, and that a measurement is made (over $t, x, y$ ) at $z=z_{2}$, which measurement partly "collapses" the wave function there. The input at $z_{2}$ to both boxes can change as a result of the acquired information, leading in turn to a change in the overall output at $z_{1}$ and $z_{3}$, and, due to reflections, a change in the wave function at $z_{2}$ at which the measurement is made. There is therefore a problem of consistency, 
in that the measurement at $z_{2}$ changes the outgoing wave function on both sides of $z_{2}$, and therefore, after a reflection, changes the ingoing wave function on both sides of $z_{2}$, that is, changes the frequency of results of the measurement at $z_{2}$, and so on. This problem seems analogous to the "grandfather paradox" (see [51, Ch. 4) of the influence of a physical system with itself between two different $t=$ constant surfaces, when reflection as well as transmission of signals along the evolution coordinate occurs. No successful attempt at analysis of this class of measurement problems is known to the author.

We reëmphasize that it is the Schrödinger equation that is taken as fundamental in the present argument, and that a theory of measurement, a probability interpretation, and an uncertainty principle, are all presumed to be derivative ideas that may require alterations from their conventional forms in order to bring them into concord with the body of formalism presented here. We have assumed that not just $\psi^{*} \psi$, but also the spatial components of the probability four-current of Sec. 2, are measurable quantities. Moreover, we assume that the flux density of a physical quantity represented by an operator $\omega$ is given by the four-current of Eqs. (33). In classical terms, the total fluxes amount to normalized counts of particles crossing a given oriented three-dimensional surface in space-time, weighted by the time of crossing or some other such quantity, and also weighted positively or negatively according as the particle crosses positively or negatively when it transits the given surface. Note that in experimental trials the particle is presumed always to transit positively across a segment of a $t=$ constant surface - Galilean geometry singles out $t=$ constant planes from all other planes in space-time and permits special treatment for these cases.

To recapitulate in other words, we assert that the evolution of a Schrödinger wave function in a spatial direction does not generally admit of description in terms of probability amplitudes. The claim is rather that the wave function in type II problems permits only the computation of certain expectation values, that is, average results of many repeated experiments with the same input signal, but such that there exists no underlying distribution of nonnegative quantities, analogous to $\psi^{*} \psi$, that accounts for the results. We advocate the non-introduction of the term "negative probabilities" as there seems to result a decrease in physical clarity thereby; instead, a kind of random behavior more general than that which can be characterized by probabilities is entailed. Khrennikov, in 52, Ch. III.2 and references given therein, describes what he calls "signed 'probabilistic' measures", which could serve as a classical analog of the local norm of Eq. (26); see also [53], §34. We infer that the conventional, probability interpretation of nonrelativistic quantum mechanics should be subordinated to an interpretation involving observable stochastic currents of particle presence, and, more comprehensively, observable stochastic currents of other physical quantities as temporal position, spatial position, energy, momentum, and so on. This "particle current" interpretation of the formalism can describe systems of both types I and II; the usual probability interpretation then applies in problems of type I and other special cases.

Although what appears to be a mathematically consistent formalism has been constructed herein, and a preliminary physical interpretation advanced, 
many questions along these lines need to be addressed, and consistency with experimental tests established, before the proposal can with confidence be regarded as a physical theory.

The above limitations notwithstanding, the formalism proposed herein has obtained results that agree to an extent with some special results previously derived, and has secured results that would be difficult to obtain by other published methods of analysis: for example, a generic expression Eqs. (88)-(93)

for the average dwell time for a particle reflecting from or passing through a time-dependent barrier.

\section{References}

[1] J. von Neumann. Mathematische Grundlagen der Quantenmechanik. Springer, Berlin, Germany, 1932.

[2] J. von Neumann. Mathematical Foundations of Quantum Mechanics. Princeton U. Pr., Princeton, NJ, USA, 1955. (English translation of Ref. 1].

[3] W. Pauli. Allgemeine prinzipien der wellenmechanik. volume XXIV, Part 1 of Handbuch der Physik. Springer, Berlin, Germany, 1933.

[4] W. Pauli. General Principles of Quantum Mechanics. Springer-Verlag, Berlin, Germany, 1980. (English translation of Ref. [3]).

[5] A. Peres. Quantum Theory: Concepts and Methods. Kluwer Academic, Dordrecht, The Netherlands, 1995.

[6] R. Omnès. The Interpretation of Quantum Mechanics. Princeton U. Press, Princeton, NJ, USA, 1994.

[7] J. J. Sakurai. Modern Quantum Mechanics. Addison-Wesley, Reading, MA, USA, revised edition, 1994.

[8] F. Capasso, K. Mohammed, and A. Y. Cho. IEEE J. Quant. Electr., QE$22: 1853,1986$.

[9] D. K. Ferry. Transport in nanostructures. Cambridge U. Pr., Cambridge, UK, 1997.

[10] M. Jonson. In D. K. Ferry and C. Jacoboni, editors, Quantum Transport in Semiconductors, chapter 10. Plenum, New York, NY, 1992.

[11] A. P. Jauho. In D. K. Ferry and C. Jacoboni, editors, Quantum Transport in Semiconductors, chapter 9. Plenum, New York, NY, 1992.

[12] A. P. Jauho. In J. Shah, editor, Hot Carriers in Semiconductor Nanostructures, chapter II.4. Academic, Boston, MA, 1992. 
[13] H. Mizuta and T. Tanoue. The Physics and applications of resonant tunnelling diodes. Cambridge U. Press, Cambridge, UK, 1995.

[14] E. H. Hauge and J. A. Støvening. Rev. Mod. Phys., 61:917, 1989.

[15] V. S. Olkhovsky and E. Recami. Phys. Reports, 214:339, 1991.

[16] R. Landauer and Th. Martin. Rev. Mod. Phys., 66:217, 1994.

[17] R. Y. Chiao and A. M. Steinberg. Progress in Optics, XXXVII:345, 1997.

[18] D. Mugnai, A. Ranfagni, and L. S. Schulman, editors. Tunneling and its implications. World Scientific, River Edge, NJ, 1997.

[19] J. A. Damborenea, I. L. Egusquiza, G. C. Hegerfeldt, and J. G. Muga. Phys. Rev. A, 66:052104, 2002.

[20] J. G. Muga, R. S. Mayato, and I. L. Egusquiza, editors. Time in Quantum Mechanics. Lecture Notes in Physics M72. Springer, Berlin, Germany, 2002.

[21] C. Lanczos. The Variational Principles of Mechanics. Dover, New York, NY, USA, 4th edition, 1986.

[22] A. Friedman. Partial differential equations of parabolic type. Prentice-Hall, Englewood Cliffs, NJ, 1964.

[23] I. Gohberg, P. Lancaster, and L. Rodman. Matrices and Indefinite Scalar Products. Birkhäuser, Basel, Switzerland, 1983.

[24] J. Kijowski. Rep. Math. Phys., 6:361, 1974.

[25] C. Piron. C. R. Acad. Sc. Paris, A286:713, 1978.

[26] B. Mielnik. Found. Phys., 24:1113, 1994.

[27] G. E. Hahne. J. Phys. A, 35:7101, 2002.

[28] L. I. Schiff. Quantum Mechanics. McGraw-Hill, New York, NY, USA, 3rd edition, 1968.

[29] H. Goldstein. Classical Mechanics. Addison Wesley, Reading, MA, USA, 2nd edition, 1980.

[30] W. H. Miller. In D. C. Clary, editor, The Theory of Chemical Reaction Dynamics, page 27. Dordrecht, The Netherlands, 1986.

[31] J. G. Muga. Characteristic times in one-dimensional scattering. In J. G. Muga, R. S. Mayato, and I. L. Egusquiza, editors, Time in Quantum Mechanics, Lecture Notes in Physics M72, chapter 2. Springer, Berlin, Germany, 2002.

[32] H. M. Nussenzweig. Phys. Rev. A, 62:042107, 2000. 
[33] W. Jaworski and D. M. Wardlaw. Phys. Rev. A, 37:2843, 1988.

[34] W. Jaworski and D. M. Wardlaw. Phys. Rev. A, 38:5404, 1988.

[35] W. Jaworski and D. M. Wardlaw. Phys. Rev. A, 40:6210, 1989.

[36] W. Jaworski and D. M. Wardlaw. Phys. Rev. A, 45:292, 1992.

[37] W. Heisenberg. Nucl. Phys., 4:532, 1957.

[38] K. L. Nagy. State Vector Spaces with Indefinite Metric in Quantum Field Theory. P. Noordhoff, Groningen, The Netherlands, 1966.

[39] S. MacLane and G. Birkhoff. Algebra. Chelsea, New York, NY, USA, 3rd edition, 1988.

[40] M. L. Goldberger and K. M. Watson. Collision Theory. J. Wiley, New York, NY, USA, 1964.

[41] F. T. Smith. Phys. Rev., 118:349-356, 1960.

[42] F. T. Smith. Phys. Rev., 119:2098, 1960.

[43] C. R. Leavens. Tunneling and its implications. World Scientific, River Edge, NJ, 1997.

[44] J. G. Muga, S. Brouard, and R. Sala. Phys. Lett. A, 167:24, 1992.

[45] S. Brouard, R. Sala, and J. G. Muga. Phys. Rev. A, 49:4312, 1994.

[46] V. S. Olkhovsky, E. Recami, and J. Jakiel. online arXiv:quant-ph/0102007, 2001.

[47] P. Busch. The time-energy uncertainty relation. In J. G. Muga, R. S. Mayato, and I. L. Egusquiza, editors, Time in Quantum Mechanics, Lecture Notes in Physics M72, chapter 3. Springer, Berlin, Germany, 2002.

[48] J. Kijowski. Phys. Rev. A, 59:897, 1999.

[49] J. G. Muga, R. S. Mayato, and I. L. Egusquiza. In J. G. Muga, R. S. Mayato, and I. L. Egusquiza, editors, Time in Quantum Mechanics, Lecture Notes in Physics M72, chapter 1. Springer, Berlin, Germany, 2002.

[50] I. L. Egusquiza, J. G. Muga, and A. D. Baute. "Standard" quantum mechanical approach to times of arrival. In J. G. Muga, R. S. Mayato, and I. L. Egusquiza, editors, Time in Quantum Mechanics, Lecture Notes in Physics M72, chapter 10. Springer, Berlin, Germany, 2002.

[51] P. J. Nahin. Time machines. Springer, New York, NY, 2nd edition, 1999.

[52] A. Khrennikov. Interpretations of Probability. VSP, Utrecht, The Netherlands, 1999.

[53] A. N. Kolmogorov and S. V. Fomin. Introductory Real Analysis. PrenticeHall, Englewood Cliffs, NJ, USA, 1970. 